\title{
Finite-element model for phase-change recording
}

\author{
J. H. Brusche and A. Segal \\ Department of Applied Mathematical Analysis, Delft University of Technology, 2628 CD Delft, The Netherlands
}

\author{
H. P. Urbach
}

Philips Research Laboratories, Professor Holstlaan 4, 5656 AA Eindhoven, The Netherlands

Received May 20, 2004; revised manuscript received October 5, 2004; accepted October 7, 2004

\begin{abstract}
The finite-element method is applied to model phase-change recording in a grooved recording stack. A rigorous model for the scattering of a three-dimensional focused spot by a one-dimensional periodic grating is used to determine the absorbed light in a three-dimensional region inside the phase-change layer. The optical model is combined with a three-dimensional thermal model to compute the temperature distribution. Land and groove recording and polarization dependence are studied, and the model is applied to the Blu-ray Disc. (C) 2005 Optical Society of America
\end{abstract}

OCIS codes: $050.1950,210.0210,210.4590,210.4770$.

\section{INTRODUCTION}

For rewritable optical storage media such as the rewritable compact disc, the rewritable digital versatile disc, and the Blu-ray Disc, data storage is based on two distinct states of a phase-change (PC) material. By application of short high-power pulses with a laser beam that is focused on the storage layer, submicrometer-sized amorphous marks are formed in a crystalline background. After the marks have been written, they can be detected by monitoring intensity variations in the reflected light of the same focused laser beam at a much lower power level.

To increase the storage capacity and the data transfer rates of $\mathrm{PC}$ optical disks, modeling of the recording process is important. By use of numerical simulations, insight can be gained into the effects of the polarization of the laser spot, the effects of the spot size and the spot shape on the mark formation, and the differences between land recording and groove recording. Furthermore, the groove geometry and the stack can be optimized. Other phenomena that can be studied by modeling are the occurrence of optical and thermal crosstrack cross talk.

The model of the recording process for PC optical disks can be divided into three parts. In the first part the electromagnetic (EM) field distribution is calculated within the disk. From this field the light absorption in the medium can be derived. It is in general assumed that all of the absorbed light is converted into heat. Then, in the second part, the temperature distribution in the medium is computed by solving the heat equation. The third part is the modeling of temperature-induced mark formation or erasure in the PC layer. In this paper only the first two parts of the model will be considered.

Because the feature sizes of the grooved structure of the medium are of the order of the wavelength of the laser spot, the polarization of the incident light cannot be neglected. A rigorous vector diffraction model based on Maxwell's equations is thus required in the calculation of the light intensity in the stack.
Many vector diffraction models have been considered by other authors to derive the EM field distribution in grooved multilayered stacks. These methods have been developed in the context of the optical readout of prerecorded digital versatile discs. Judkins and Ziolkowski ${ }^{1}$ used the finite-difference time-domain method with absorbing boundary conditions and with a Lorentz dispersion model to incorporate conducting metals of which the real part of the electric permittivity is negative. Liu and Kowarz ${ }^{2}$ applied the finite-difference frequency-domain method by using Higdon's absorbing boundary conditions and a preconditioned conjugate gradient method to solve the discretized system of equations. Marx and Psaltis ${ }^{3}$ used a Lippmann-Schwinger-type of integral equation to solve the diffraction problem for a two-dimensional (2D) incident beam.

In the above-mentioned papers the authors considered the interaction of a 2D incident beam with $2 \mathrm{D}$ grooved geometries. Yeh et $a l .{ }^{4}$ studied the more general problem of a three-dimensional (3D) spot that is incident on a $2 \mathrm{D}$ periodically grooved structure. We will call this type of model a two-and-one-half dimensional $\left(2 \frac{1}{2} \mathrm{D}\right)$ model. The incident spot is expanded into plane waves, and a scattering problem is solved for every incident plane wave by use of a coordinate transformation to transfer the diffraction problem on the grooved structure to a configuration consisting of flat interfaces but with inhomogeneous materials (Chandezon's method).

Recently some authors published models that combine the calculation of the $3 \mathrm{D}$ EM field distribution, due to a $3 \mathrm{D}$ incident spot, with the computation of the 3D temperature distribution. Peng and Mansuripur ${ }^{5}$ used a finite-difference time-domain algorithm formulated in a $3 \mathrm{D}$ curvilinear coordinate system in order to determine the EM field distribution in a PC optical recording stack and to study thermal cross-track cross-talk effects. From the EM field, the energy that is absorbed by the medium is calculated. This absorbed energy is converted into heat and is used as the source term in the thermal diffu- 


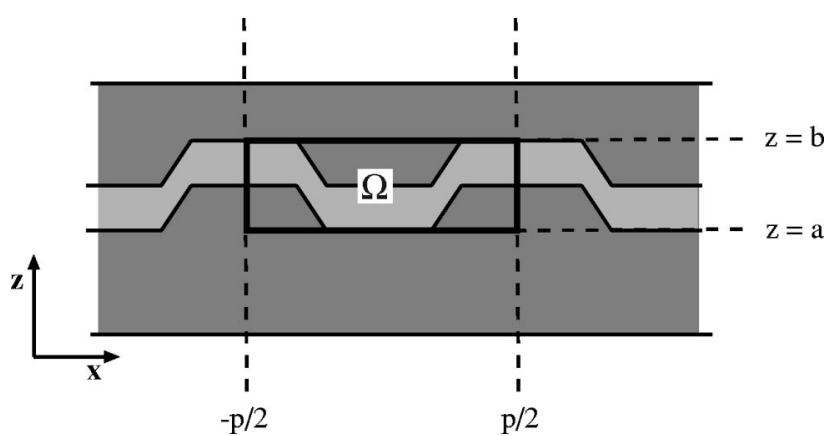

Fig. 1. Schematic visualization of the unit cell $\Omega$ in a cross section of an optical disk.

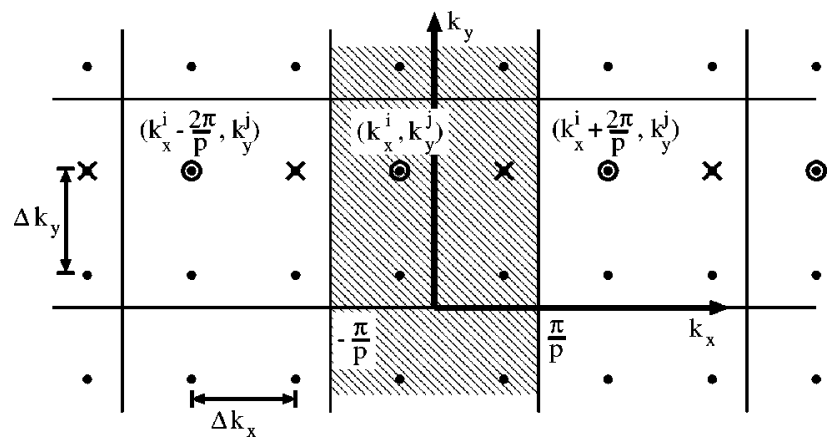

Fig. 2. Section of $\left(k_{x}, k_{y}\right)$ space showing a fragment of the Brillouin zone $\mathrm{B} \times \mathrm{R}$ (hatched). The grid distance $\Delta k_{x}$ is taken such that $2 \pi / p$ is a multiple of $\Delta k_{x}$ (in the figure $2 \pi / p=2 \Delta k_{x}$ ). For clarity, the spacing in the $k_{y}$ direction, $\Delta k_{y}$, is chosen equal to $\Delta k_{x}$. The encircled points, on the one hand, and the crossed grid points, on the other hand, are sets of grid points that are each other's order.

sion equation. To obtain the temperature distribution in the medium, Peng and Mansuripur solved this equation numerically by using the alternating-direction implicit finite-difference technique. Nishi et al. ${ }^{6}$ used a finitedifference time-domain model based on the Lorentz dispersion model published by Judkins and Ziolkowski ${ }^{1}$ to compute the 3D absorbed energy distribution.

In this paper we also present a model in which we combine an optical model and a thermal model. To calculate the EM field in a multilayered recording stack, we will use the $2 \frac{1}{2} \mathrm{D}$ model by Brok and Urbach. ${ }^{7}$ In that paper, similar to Yeh et al., ${ }^{4}$ the diffraction of a 3D incident spot on a $2 \mathrm{D}$ configuration, by means of a plane-wave expansion of the spot, was also considered. But the sampling is done such that the plane waves can be divided into sets that, for the given period of the grating, consist of waves that are orders of each other. This considerably reduces the number of scattering problems that has to be solved. The superposition of the plane waves in each set yields an incident field that is, apart from a phase shift, periodic. The interaction of this incident field with the periodic grating can be computed by solving a single boundaryvalue problem (BVP). This BVP is derived on a $2 \mathrm{D}$ computational box that is one period wide and is called a unit cell. Instead of approximative absorbing boundary conditions, exact radiation conditions for the scattered near field are used. The solution of the BVP is computed with the finite-element method (FEM). To calculate the absorbed EM energy in a 3D neighborhood of the unit cell that is illuminated by the spot and that is relevant for the heat diffusion problem, one must first extend the solution of the BVPs from the unit cell to this $3 \mathrm{D}$ region by using the property that the fields are, apart from a phase shift, periodic. After this, the extended fields are added coherently to compute the total EM field in the 3D region of interest.

In our model, both the total EM field and the temperature distribution are calculated with the FEM. All finiteelement computations are performed with the SEPRAN ${ }^{8}$ finite-element package. Besides the fact that a lot is known about its mathematical properties, the FEM has certain advantages over other methods. The main advantage is that all kinds of geometries, such as bumped layers, can be simulated. The shape of the interfaces is not restricted, and kinks are permitted. Furthermore, the presence of metals with negative real electric permittivity does not cause any problem. For configurations that are of the order of the wavelength such as in the problem studied in this paper, a relative error in the total field, measured in the energy norm, of less than $1 \%$, requires a mesh of approximately 20 elements per wavelength (measured in the material). However, when the configuration is many wavelengths large, more elements per wavelength are needed to prevent the accumulation of phase errors.

Owing to the fact that the $3 \mathrm{D}$ scattering problem is solved by expanding the incident field into fields that are periodic apart from a phase shift and by solving for each of these incident fields a $2 \mathrm{D}$ problem on the unit cell, the systems of equations to be solved are sufficiently small for a direct method (Gaussian elimination) to be applied. By the use of a direct method, it is guaranteed that the correct solution is always obtained.

In the present model of the recording process, the spot is considered to be turned on continuously for a given period of time. For a given stack geometry, the absorbed energy in the medium is derived and used as heat source in the thermal diffusion equation. Changes in the optical properties of the medium due to the heating by the spot are not taken into account.

The content of this paper is as follows. In Section 2 we consider the scattering of an arbitrary 3D incident field by a one-dimensional diffraction grating. In particular, we discuss how the solutions of the scattering problems on the unit cell are extended to the 3D region of interest for the thermal problem. In Section 3 we discuss how the acquired absorbed energy distribution, i.e., the heat source, is integrated into the 3D thermal diffusion model. We conclude this paper with a benchmark study in which we compare our model qualitatively for a stationary spot with that of Peng and Mansuripur. Finally, we consider land and groove recording for the Blu-ray Disc.

\section{SCATTERING OF A FOCUSED SPOT BY A ONE-DIMENSIONAL DIFFRACTION GRATING}

Consider a periodic grating structure as shown in Fig. 1. With respect to a Cartesian coordinate system $(x, y, z)$, the grating is assumed to be $p$ periodic in the $x$ direction 


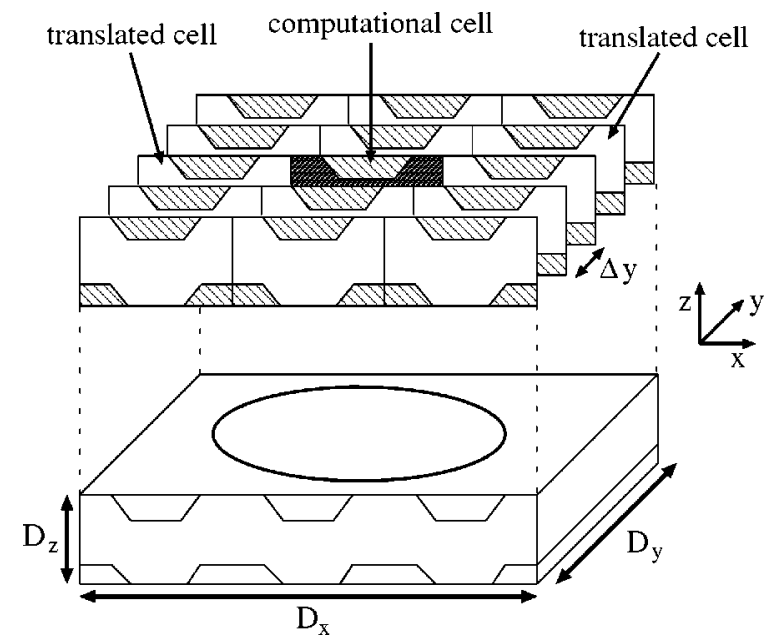

Fig. 3. Extended geometry. The cell on which the BVPs are formulated is called the computational cell. The extended region is taken large enough to contain at least the region in which the intensity of the laser spot is essentially nonzero.

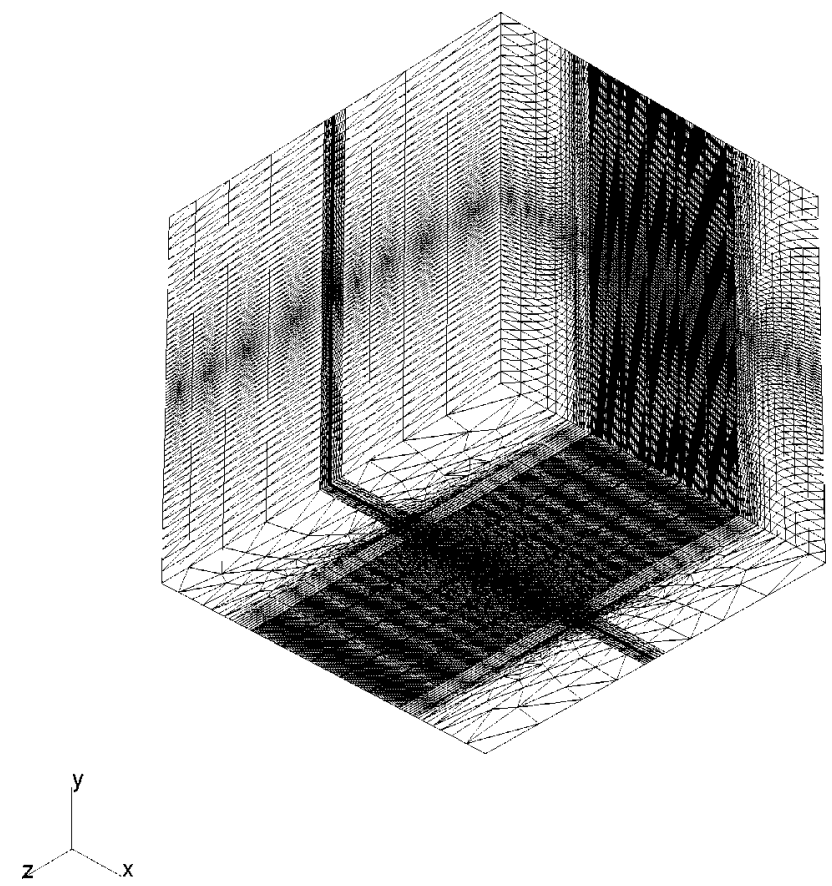

Fig. 4. Tetrahedral mesh used in the thermal calculations. In the region in which the absorbed energy is mapped, the grid size is taken of the same order in size as that of the triangular mesh of the optical model.

and translation invariant parallel to the $y$ axis. The $z$ axis coincides with the optical axis of the illumination system. All materials are nonmagnetic; hence the magnetic permeability $\mu=\mu_{0}$ everywhere. Let the $2 \mathrm{D}$ unit cell $\Omega$ be the region in the plane $y=0$ defined by

$$
\Omega=\left\{(x, z) \mid-\frac{p}{2}<x<\frac{p}{2}, a<z<b\right\} .
$$

The planes $z=a$ and $z=b$ are chosen such that $z<a$ and $z>b$ are half-spaces in which the electric permittivity $\epsilon$ is constant and such that $\Omega$ has minimal area.

Let the electric field $\mathbf{E}^{i}$ of the incident spot be given in a certain plane $z=z_{i}$ above the grating. Let
$\mathcal{F}\left(\mathbf{E}^{i}\right)\left(k_{x}, k_{y}, z_{i}\right)$ be the Fourier transform of $\mathbf{E}^{i}$ with respect to $x$ and $y$ in the plane $z=z_{i}$ :

$$
\begin{aligned}
\mathcal{F}\left(\mathbf{E}^{i}\right)\left(k_{x}, k_{y}, z_{i}\right)= & \int_{-\infty}^{\infty} \int_{-\infty}^{\infty} \mathbf{E}^{i}\left(x, y, z_{i}\right) \\
& \times \exp \left[-i\left(k_{x} x+k_{y} y\right)\right] \mathrm{d} x \mathrm{~d} y .
\end{aligned}
$$

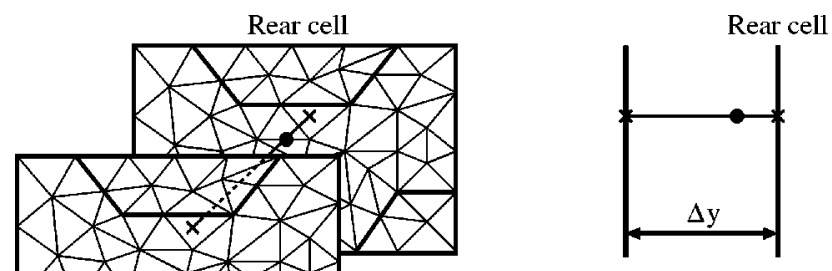

Front cell

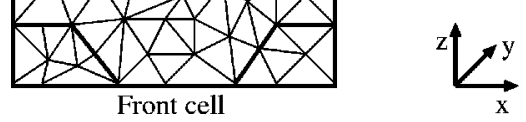

Fig. 5. To compute the absorbed energy in a nodal point (circles) of the tetrahedral mesh for the thermal model, we first calculate the values in the intersections $X$ with the two enclosing expanded cells of the optical model. These values are linearly interpolated by using the piecewise linear basis functions on the triangles. The absorbed energy in the nodal point is then the linearly interpolated value of the values in the intersection points.

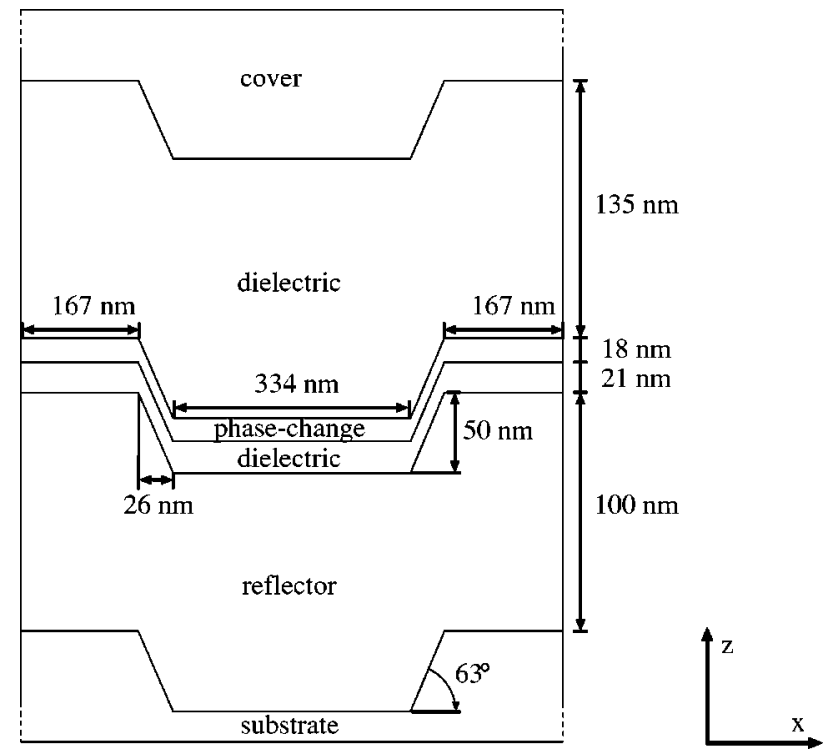

Fig. 6. Cross section of the stack as used in configuration $\mathrm{C} 1 \mathrm{ac}-$ cording to Peng and Mansuripur. ${ }^{5}$ The structure is translation invariant in the $y$ direction.

Table 1. Numerical Values for the Complex Refractive Index $n$, Heat Capacity $\rho C$, and Thermal Conductivity $\kappa$ as Used in Simulations for the Configuration of Fig. 6

\begin{tabular}{lccc}
\hline Material & $n$ & $\begin{array}{c}\rho C \\
\left(\mathrm{~J} / \mathrm{cm}^{3}{ }^{\circ} \mathrm{C}\right)\end{array}$ & $\begin{array}{c}\kappa \\
\left(\mathrm{W} / \mathrm{cm}{ }^{\circ} \mathrm{C}\right)\end{array}$ \\
\hline Cover (polycarbonate) & 1.54 & 1.7 & 0.0023 \\
Dielectric $\left(\mathrm{ZnS}_{-} \mathrm{SiO}_{2}\right)$ & 2.3 & 2.005 & 0.0058 \\
PC layer $\left(\mathrm{Ge}_{2} \mathrm{Sb}_{2.3} \mathrm{Te}_{5}\right)$ & $1.78+3.23 i$ & 1.285 & 0.006 \\
Reflector $(\mathrm{Al} \mathrm{alloy})$ & $0.5+3.8 i$ & 2.45 & 0.2 \\
Substrate (polycarbonate) & 1.54 & 1.7 & 0.0023 \\
\hline
\end{tabular}




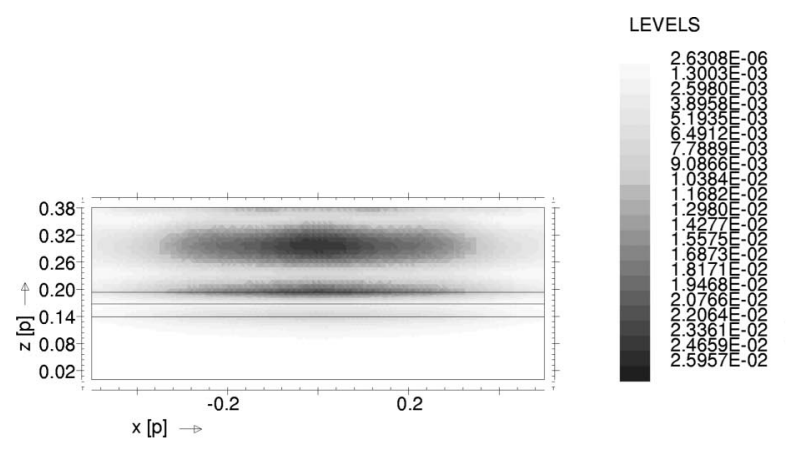

(a)

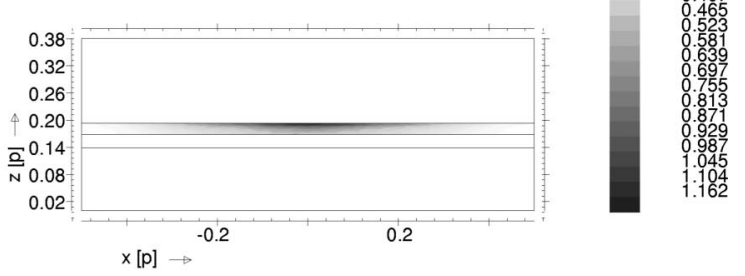

(b)

Fig. 7. Dominant field components (arbitrary units) and absorbed energy (W/ $p^{3}$ ) in the unit cell for a TM-polarized spot that is incident on a flat version of the multilayered stack of Fig. 6. (a) $\left|H_{y}\right|$ and (b) absorbed energy. The spot is focused in air in the middle of the PC layer.

The inverse Fourier transform yields

$$
\begin{aligned}
\mathbf{E}^{i}\left(x, y, z_{i}\right)= & \frac{1}{4 \pi^{2}} \int_{-\infty}^{\infty} \int_{-\infty}^{\infty} \mathcal{F}\left(\mathbf{E}^{i}\right)\left(k_{x}, k_{y}, z_{i}\right) \\
& \times \exp \left[i\left(k_{x} x+k_{y} y\right)\right] \mathrm{d} k_{x} \mathrm{~d} k_{y},
\end{aligned}
$$

which corresponds to a plane-wave expansion of the incident field with wave vectors $\mathbf{k}$, of which $k_{x}$ and $k_{y}$ are the components along the $x$ and $y$ axes, respectively. The time dependence is given by the factor $\exp (-i \omega t)$, which is omitted from all formulas.

We will always choose the branch of the complex square root $z^{1 / 2}$ such that the cut is along the negative real axis and such that, for positive real $z, z^{1 / 2}>0$ and $(-z)^{1 / 2}$ $=+i z^{1 / 2}$. Because all waves of the incident field are propagating downward and since the time dependence of the fields is assumed to be given by $\exp (-i \omega t)$, it follows that the $z$ component of the wave vectors $\mathbf{k}$ in the planewave expansion of the incident field is given by $k_{z}$ $=-\left[\omega^{2} \epsilon_{0} \mu_{0} \epsilon_{u}-\left(k_{x}^{2}+k_{y}^{2}\right)\right]^{1 / 2}$, where $\epsilon_{u}$ is the relative electric permittivity of the material in the upper homogeneous half-space $z>b$. The incident field in any other plane $z=$ constant is thus given by

$$
\begin{aligned}
\mathbf{E}^{i}(x, y, z)= & \frac{1}{4 \pi^{2}} \int_{-\infty}^{\infty} \int_{-\infty}^{\infty} \mathcal{F}\left(\mathbf{E}^{i}\right)\left(k_{x}, k_{y}, z_{i}\right) \\
& \times \exp \left\{i\left[k_{x} x+k_{y} y+k_{z}\left(z-z_{i}\right)\right]\right\} \mathrm{d} k_{x} \mathrm{~d} k_{y} .
\end{aligned}
$$

We rewrite this as follows:

$$
\begin{aligned}
\mathbf{E}^{i}(x, y, z) \\
=\frac{1}{4 \pi^{2}} \int_{-\infty}^{\infty} \int_{-\infty}^{\infty} \mathcal{F}\left(\mathbf{E}^{i}\right)\left(k_{x}, k_{y}, z_{i}\right) \\
\quad \times \exp \left\{i\left[k_{x} x+k_{y} y+k_{z}\left(z-z_{i}\right)\right]\right\} \mathrm{d} k_{x} \mathrm{~d} k_{y} \\
=\frac{1}{4 \pi^{2}} \sum_{m=-\infty}^{\infty} \int_{-\infty}^{\infty} \int_{-\pi / p+2 \pi m / p}^{\pi / p+2 \pi m / p} \mathcal{F}\left(\mathbf{E}^{i}\right)\left(k_{x}, k_{y}, z_{i}\right) \\
\quad \times \exp \left\{i\left[k_{x} x+k_{y} y+k_{z}\left(z-z_{i}\right)\right]\right\} \mathrm{d} k_{x} \mathrm{~d} k_{y} \\
=\frac{1}{4 \pi^{2}} \sum_{m=-\infty}^{\infty} \int_{-\infty}^{\infty} \int_{-\pi / p}^{\pi / p} \mathcal{F}\left(\mathbf{E}^{i}\right)\left(k_{x}+\frac{2 \pi m}{p}, k_{y}, z_{i}\right) \\
\quad \times \exp \left\{i\left(k_{x}+\frac{2 \pi m}{p}\right) x+i k_{y} y\right. \\
\left.\left.\quad+k_{z}^{m}\left(z-z_{i}\right)\right]\right\} \mathrm{d} k_{x} \mathrm{~d} k_{y} \\
=\int_{-\infty}^{\infty} \int_{-\pi / p}^{\pi / p} \mathbf{E}_{k_{x}, k_{y}}(x, z) \exp \left[i\left(k_{x} x+k_{y} y\right)\right] \mathrm{d} k_{x} \mathrm{~d} k_{y},
\end{aligned}
$$

where

$$
\begin{aligned}
k_{z}^{m} & =-\left[\omega^{2} \epsilon_{0} \epsilon_{u} \mu_{0}-\left(k_{x}+2 \pi m / p\right)^{2}-k_{y}{ }^{2}\right]^{1 / 2}, \\
\mathbf{E}_{k_{x}, k_{y}}^{i}(x, z) \exp \left[i\left(k_{x} x+k_{y} y\right)\right] & \\
= & \frac{1}{4 \pi^{2}} \sum_{m=-\infty}^{\infty} \mathcal{F}\left(\mathbf{E}^{i}\right)\left(k_{x}+\frac{2 \pi m}{p}, k_{y}, z_{i}\right) \\
& \times \exp \left[i\left(k_{x}+\frac{2 \pi m}{p}\right) x+i k_{y} y+i k_{z}^{m}\left(z-z_{i}\right)\right] .
\end{aligned}
$$

The field $\mathbf{E}_{k_{x}, k_{y}}^{i}(x, z)$ is $p$ periodic with respect to $x$ and is independent of $y$. The field (6) depends harmonically on $y$ and is, apart from a phase-shift, $p$ periodic in $x$. 
Since the incident field satisfies the source-free Maxwell equations in the upper half-space,

$$
\begin{aligned}
& \boldsymbol{\nabla} \times \mathbf{E}^{i}=i \omega \mu_{0} \mathbf{H}^{i}, \\
& \boldsymbol{\nabla} \times \mathbf{H}^{i}=-i \omega \epsilon_{0} \epsilon_{u} \mathbf{E}^{i},
\end{aligned}
$$

we have

$$
\mathcal{F}\left(\mathbf{H}^{i}\right)\left(k_{x}, k_{y}, z\right)=\frac{\mathbf{k}}{\omega \mu_{0}} \times \mathcal{F}\left(\mathbf{E}^{i}\right)\left(k_{x}, k_{y}, z\right) .
$$

For the magnetic field component of the incident spot $\mathbf{H}^{i}$, we can therefore write

$$
\begin{aligned}
\mathbf{H}^{i}(x, y, z)= & \int_{-\infty}^{\infty} \int_{-\pi / p}^{\pi / p} \mathbf{H}_{k_{x}, k_{y}}^{i}(x, z) \\
& \times \exp \left[i\left(k_{x} x+k_{y} y\right)\right] \mathrm{d} k_{x} \mathrm{~d} k_{y},
\end{aligned}
$$

where

$$
\begin{aligned}
\mathbf{H}_{k_{x}, k_{y}}^{i}(x, z) \exp \left[i\left(k_{x} x+k_{y} y\right)\right] & \\
= & \frac{1}{4 \pi^{2}} \sum_{m=-\infty}^{\infty} \frac{\mathbf{k}^{m}}{\omega \mu_{0}} \times \mathcal{F}\left(\mathbf{E}^{i}\right)\left(k_{x}+\frac{2 \pi m}{p}, k_{y}, z_{i}\right) \\
& \times \exp \left[i\left(k_{x}+\frac{2 \pi m}{p}\right) x+i k_{y} y+i k_{z}^{m}\left(z-z_{i}\right)\right] .
\end{aligned}
$$

Here $\mathbf{k}^{m}=\left(k_{x}+2 \pi m / p, k_{y}, k_{z}^{m}\right)$. We have thus written the electric and magnetic components of the incident spot as an integral over $k_{x}$ and $k_{y}$ of the fields

$$
\begin{aligned}
& \mathbf{E}_{k_{x}, k_{y}}^{i}(x, z) \exp \left[i\left(k_{x} x+k_{y} y\right)\right], \\
& \mathbf{H}_{k_{x}, k_{y}}^{i}(x, z) \exp \left[i\left(k_{x} x+k_{y} y\right)\right],
\end{aligned}
$$

where $\mathbf{E}_{k_{x}, k_{y}}^{i}(x, z)$ and $\mathbf{H}_{k_{x}, k_{y}}^{i}(x, z)$ are $p$ periodic in $x$. The integral extends over $\mathbb{B} \times \mathbb{R}$, where $\mathbb{B}$ is the onedimensional Brillouin zone, defined by

$$
\mathbb{B}=\left\{k_{x} \mid-\frac{\pi}{p}<k_{x}<\frac{\pi}{p}\right\} .
$$

Let $\epsilon(\mathbf{r})$ denote the relative (in general, complexvalued) electric permittivity at position $\mathbf{r}=(x, y, z)$. Then $\epsilon$ is $p$ periodic with respect to $x$ and independent of $y$. It then follows from Bloch's theorem that for each of the incident fields (12) and (13) the corresponding scattered fields are, apart from a phase shift, $p$ periodic in $x$ and harmonic in $y$ for the same $k_{x}$ and $k_{y}$ as the incident field. Hence the total EM field can, similarly to the incident and the scattered fields, be written as an integral:

$$
\begin{aligned}
\mathbf{E}^{\mathrm{tot}}(x, y, z)= & \int_{-\infty}^{\infty} \int_{-\pi / p}^{\pi / p} \mathbf{E}_{k_{x}, k_{y}}^{\mathrm{tot}}(x, z) \\
& \times \exp \left[i\left(k_{x} x+k_{y} y\right)\right] \mathrm{d} k_{x} \mathrm{~d} k_{y}, \\
\mathbf{H}^{\mathrm{tot}}(x, y, z)= & \int_{-\infty}^{\infty} \int_{-\pi / p}^{\pi / p} \mathbf{H}_{k_{x}, k_{y}}^{\mathrm{tot}}(x, z) \\
& \times \exp \left[i\left(k_{x} x+k_{y} y\right)\right] \mathrm{d} k_{x} \mathrm{~d} k_{y},
\end{aligned}
$$

where $\mathbf{E}_{k_{x}, k_{y}}^{\mathrm{tot}}(x, z)$ and $\mathbf{H}_{k_{x}, k_{y}}^{\mathrm{tot}}(x, z)$ are $p$ periodic in $x$ and independent of $y$.

It is shown in Ref. 7 that the field $\mathbf{E}_{k_{x}, k_{y}}^{\text {tot }}(x, z)$, $\mathbf{H}_{k_{x}, k_{y}}^{\text {tot }}(x, z)$ can be derived from the solution of a BVP on $\Omega$ with the right-hand side derived from the incident field $\mathbf{E}_{k_{x}, k_{y}}^{i}(x, z), \mathbf{H}_{k_{x}, k_{y}}^{i}(x, z)$. At interfaces between different materials across which $\epsilon$ is discontinuous, the tangential components of $\mathbf{E}_{k_{x}, k_{y}}^{\text {tot }}$ and $\mathbf{H}_{k_{x}, k_{y}}^{\text {tot }}$ are continuous. For every $\left(k_{x}, k_{y}\right)$, the corresponding BVP consists of a coupled system of two linear second-order partial differential equations in terms of the axial components $\hat{\boldsymbol{i}}_{y} \cdot \mathbf{E}_{k_{x}, k_{y}}^{\mathrm{tot}}$ and $\hat{\boldsymbol{i}}_{y} \cdot \mathbf{H}_{k_{x}, k_{y}}^{\mathrm{tot}}$ only, with exact radiation conditions on the lower and upper boundaries $z=a$ and $z$ $=b$, respectively, and two periodic boundary conditions on the boundaries $x= \pm p / 2$. This BVP is equivalent to the Maxwell equations for the given $\left(k_{x}, k_{y}\right)$. The radiation boundary conditions are derived with a plane-wave expansion of the reflected and the total fields in the upper and lower half-spaces. These boundary conditions are nonlocal, since they are formulated in terms of Fourier series. The series have infinitely many terms because they contain contributions not only of the propagating plane waves but also of the evanescent waves. The series converge slowly so that a direct numerical summation would be time-consuming. However, as has been explained in Ref. 9, the general term of the series can be expanded asymptotically in terms of inverse powers of the Fourier in$\operatorname{dex} m$, and the sum of the series that is thus obtained can be summed analytically. The series whose terms consist of the remainders of the asymptotic expansion still have to be summed numerically, but these series converge much faster than the original series. In this way, a very efficient implementation of the radiation boundary conditions is obtained.

We remark here that the expressions given in Brok and Urbach $^{7}$ for the nonlocal boundary conditions are correct only if the dielectric permittivity is continuous across the upper and lower boundaries of the computational domain. But in the more general case that the computational domain is chosen such that $\epsilon$ is different on both sides of these boundaries, the expressions should actually be slightly modified. In the computer code that was used to obtain the results described in Brok and Urbach ${ }^{7}$ and in the present paper, the correct expressions were implemented.

The advantage of the formulation in terms of the axial field components $\hat{\boldsymbol{i}}_{y} \cdot \mathbf{E}_{k_{x}, k_{y}}^{\mathrm{tot}}$ and $\hat{\boldsymbol{i}}_{y} \cdot \mathbf{H}_{k_{x}, k_{y}}^{\text {tot }}$ is that these components are always tangential to all interfaces; hence they are continuous everywhere. Therefore, in the FEM, one can use standard (so-called $H^{1}$ ) linear elements. A drawback of this approach is the loss of accuracy when the other field components are computed, since they must be obtained by differentiation of the axial components.

The incident field is computed with the package DIFFRACT ${ }^{10}$ for a TE- or a TM-polarized plane wave or a Gaussian beam that is focused by a positive lens of high numerical aperture (NA). TE means that the electric field of the plane wave or Gaussian beam that is incident on the lens is parallel to the grooves, and TM means that the magnetic field is parallel to the grooves. In the calculation of the field in the focal plane $z=z_{i}$, the rotation 


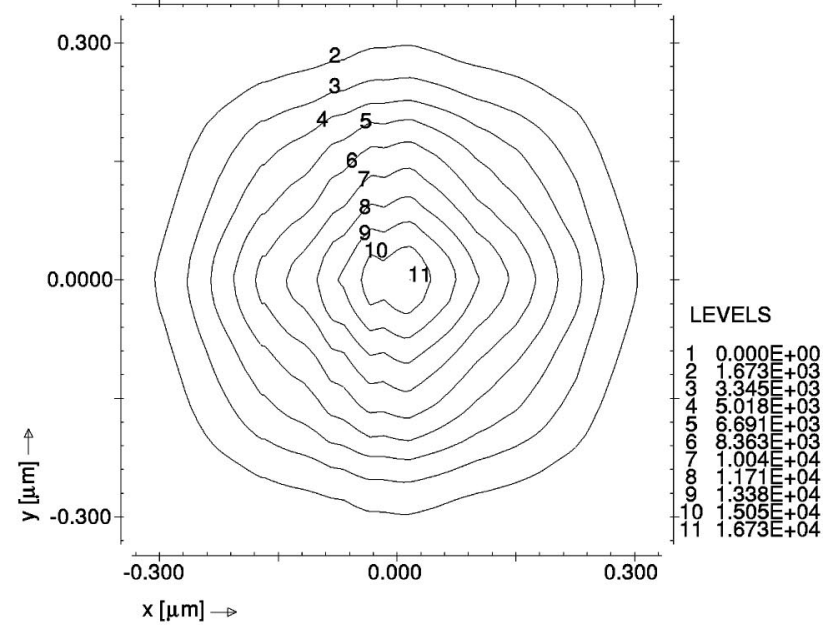

(b)

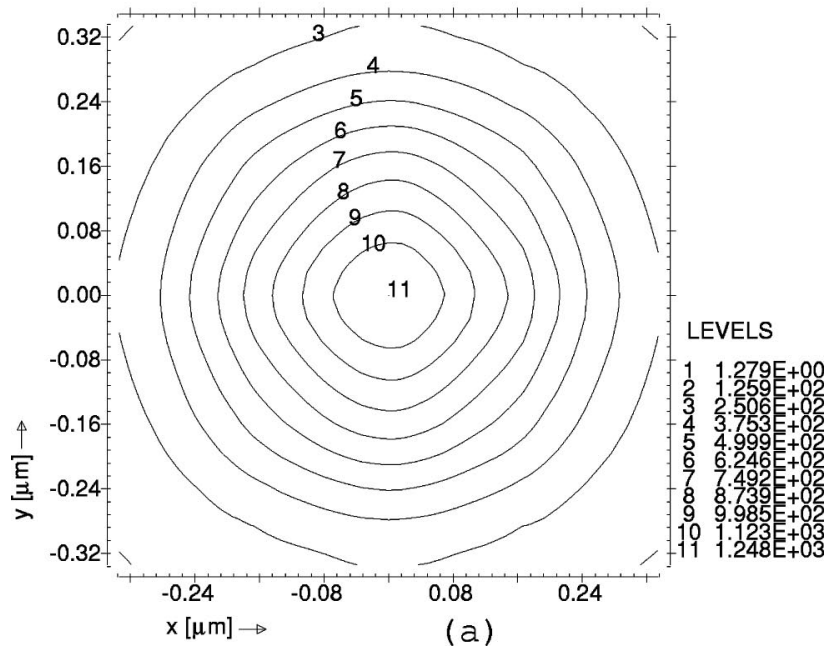

Fig. 8. Top view of the absorbed energy $\left(10^{4} \mathrm{~W} / \mu \mathrm{m}^{3}\right)$ and the temperature distribution [degrees Celsius $\left({ }^{\circ} \mathrm{C}\right)$ ] halfway in the PC layer for a TM-polarized spot incident on a flat version of the stack shown in Fig. 6. (a) Absorbed energy, (b) temperature distribution.

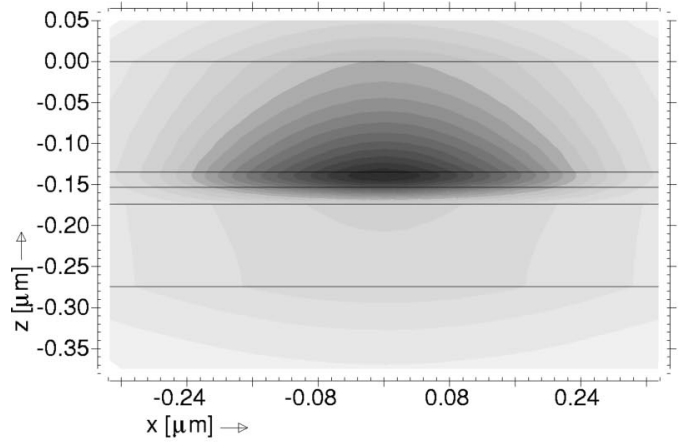

(a) of the field vectors is taken into account by use of the theory of Ignatowsky ${ }^{11}$ and Richards and Wolf. ${ }^{12}$ The focused spot therefore is not perfectly TE or TM polarized but is only predominantly TE or TM polarized. DIFFRACT gives only the $E_{x}^{i}$ and $E_{y}^{i}$ components of the incident field, whereas to solve our BVPs we need the $E_{y}^{i}$ and $H_{y}^{i}$ components. Both $E_{x}^{i}$ and $E_{y}^{i}$ can be written as a superposition of plane waves in the plane $z=z_{i}$, as in Eq. (3). It then follows from $\mathcal{F}\left(\mathbf{E}^{i}\right) \cdot \mathbf{k}=0$ that

$$
\mathcal{F}\left(E_{z}^{i}\right)=-\frac{1}{k_{z}}\left[k_{x} \mathcal{F}\left(E_{x}^{i}\right)+k_{y} \mathcal{F}\left(E_{y}^{i}\right)\right] .
$$

The magnetic incident field $\mathbf{H}^{i}$ and, in particular, $H_{y}^{i}$ are then found with Faraday's law [Eq. (7)].

The double integrals (5) and (10) with $z=z_{i}$ are discretized on a grid $\left(k_{x}^{i}, k_{y}^{j}\right)$ in $\mathrm{B} \times \mathbb{R}$. The fast Fourier transform (FFT) is used to compute the amplitudes $\mathcal{F}\left(\mathbf{E}^{i}\right)\left(k_{x}^{i}, k_{y}^{j}, z_{i}\right)$. We choose the mesh distance $\Delta k_{x}$ of the FFT grid such that $2 \pi / p$ is a multiple of $\Delta k_{x}$, i.e., such that

$$
2 \pi / p=\nu \Delta k_{x},
$$

for some integer $\nu$. Then, for every grid point $\left(k_{x}^{i}, k_{y}^{j}\right)$ that is in $\mathrm{B} \times \mathrm{R}$ and with $k_{z}^{i}=i \Delta k_{z}$, we find that the points that are the higher orders of $\left(k_{x}^{i}, k_{y}^{j}\right)$ can be written as

$$
\left(k_{x}^{i}+\frac{2 \pi m}{p}, k_{y}^{j}\right)=\left(k_{x}^{\nu m}, k_{y}^{j}\right),
$$

where $m$ is an integer (see Fig. 2). We conclude that, owing to the fact that $\Delta k_{x}$ is chosen such that $2 \pi / p$ is a multiple of $\Delta k_{x}$, the higher orders of a grid of the FFT grid that is in $B \times \mathbb{R}$ are also in the FFT grid. Therefore the FFT grid of the spot is divided into the following sets:

$$
\left\{\left(k_{x}^{i}+\frac{2 \pi m}{p}, k_{y}^{j}\right), m \text { integer }\right\},
$$

where $\left(k_{x}^{i}, k_{y}^{j}\right)$ is a grid point that is inside $\mathbb{B} \times \mathbb{R}$. The sum of plane waves in each set is, apart from a phase
LEVELS

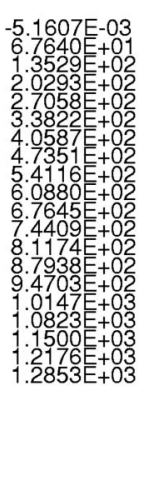

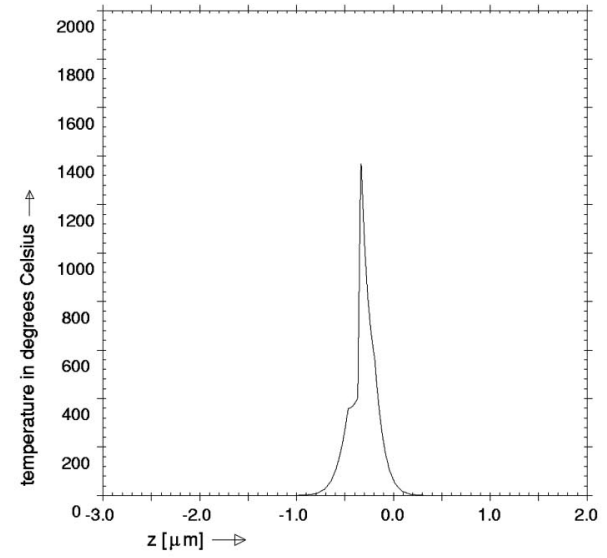

(b)

Fig. 9. Temperature distribution $\left({ }^{\circ} \mathrm{C}\right)$ for a TM-polarized spot (a) in a cross section perpendicular to the groove direction and (b) a cross section of (a) perpendicular to the interfaces along the optical axis. The stack is that of Fig. 6 except that the interfaces are flat. The temperature values are much too large because the effect of latent heat is neglected, but the shape of the cross sections should be correct. 
shift, $p$ periodic in $x$, and therefore this sum can be taken as an incident field for the BVP corresponding to $\left(k_{x}^{i}, k_{j}^{j}\right)$. In this way the number of BVPs that have to be solved is minimum and equal to the number of grid points of the FFT grid that are inside the set $\mathbb{B} \times \mathbb{R}$.

For every pair $\left(k_{x}^{i}, k_{y}^{j}\right)$ in $\mathbb{B} \times \mathbb{R}$, the BVP is solved with the FEM. A triangular grid is generated for the unit cell, and piecewise linear basis functions are used for the interpolation of the solution. For sufficient accuracy, around twenty mesh points per wavelength (measured in the material) are taken. The resulting large matrix is complex valued and, in general, nonsymmetric and nonHermitian and is partially nonsparse owing to the nonlocal boundary conditions at $z=a$ and $z=b$. Because the matrix is relatively small, a direct solver is used.

The formulas (15) and (16) for the total EM field do not only apply to points in the unit cell but also are valid for arbitrary $(x, y, z)$. For all positive and negative integers $\mu, \nu$, the total EM field in a strip $\{(x, y, z) \mid(\mu$ $\left.\left.-\frac{1}{2}\right) p \leqslant x \leqslant\left(\mu+\frac{1}{2}\right) p ; y=\nu \Delta y\right\}$ is given by

$$
\begin{aligned}
\mathbf{E}^{\mathrm{tot}}(\bar{x}+\mu p, y, z)= & \int_{-\infty}^{\infty} \int_{-\pi / p}^{\pi / p} \mathbf{E}_{k_{x}, k_{y}}^{\mathrm{tot}}(\bar{x}, z) \exp \left[i k_{x}(\bar{x}\right. \\
& \left.+\mu p)+i k_{y} \nu \Delta y\right] \mathrm{d} k_{x} \mathrm{~d} k_{y}, \\
\mathbf{H}^{\mathrm{tot}}(\bar{x}+\mu p, y, z)= & \int_{-\infty}^{\infty} \int_{-\pi / p}^{\pi / p} \mathbf{H}_{k_{x}, k_{y}}^{\mathrm{tot}}(\bar{x}, z) \exp \left[i k_{x}(\bar{x}\right. \\
& \left.+\mu p)+i k_{y} \nu \Delta y\right] \mathrm{d} k_{x} \mathrm{~d} k_{y},
\end{aligned}
$$

where $-p / 2<\bar{x}<p / 2$. Hence the extension of the total EM field from the unit cell to other cells in the plane $y$ $=0$ and in other planes $y=\nu \Delta y$ requires that the fields corresponding to every $\left(k_{x}, k_{y}\right)$ in $\mathbb{B} \times \mathbb{R}$ are multiplied by the factor $\exp \left[i\left(k_{x} \mu p+k_{y} \nu \Delta y\right)\right]$ for appropriate integers $\mu$ and $\nu$. Subsequently, the integrals (15) and (16) over $\mathrm{B} \times \mathbb{R}$ have to be computed. Note that owing to the $\left(k_{x}, k_{y}\right)$ dependency of the exponential multiplication factor, the extension to a $3 \mathrm{D}$ region of interest cannot be done after the total EM field in the unit cell has been calculated. Instead, each periodic solution must be extended separately, and after that the integral must be computed.

To be more specific, the following procedure has been implemented. The total EM field for the unit cell is extended to a region $\Lambda_{\text {opt }}$ with dimensions $\left(D_{x}, D_{y}, D_{z}\right.$ $=b-a)$ that are so large that outside of $\Lambda_{\text {opt }}$ the spot can be considered to be negligible. For the discretization in the $y$ direction, a sufficiently small step size $\Delta y$ is chosen; see Fig. 3. The unit cell on which the BVP is formulated will from this point onward be referred to as the computational cell. For every pair $\left(k_{x}^{i}, k_{y}^{j}\right)$ in $\mathbb{B} \times \mathbb{R}$, the corresponding BVP is solved on the computational cell with an incident field $\mathbf{E}_{k_{x}^{i}, k_{y}^{j}}^{i}(x, z), \mathbf{H}_{k_{x}^{i}, k_{y}^{j}}^{i}(x, z)$. For fixed $k_{y}^{j}$ the solutions corresponding to all $k_{x}^{i^{y}}$ are extended to the neighboring cells that are in the same plane $y$ $=0$ as the computational cell by means of multiplication by the appropriate factor $\exp \left(i k_{x}^{i} \mu p\right)$, after which the extended fields are added coherently. The thus obtained accumulated field in the plane $y=0$ corresponding to the fixed $k_{y}^{j}$ is then extended to the cells in the $y$ direction by

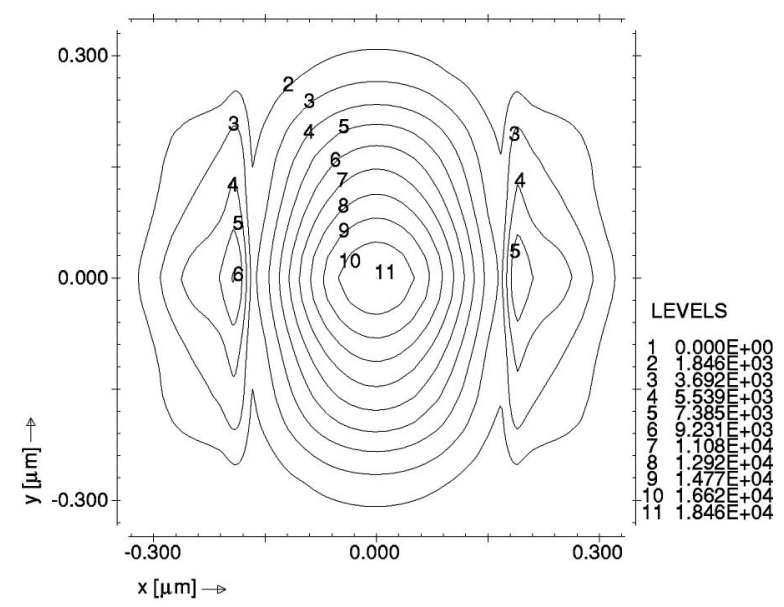

(a)

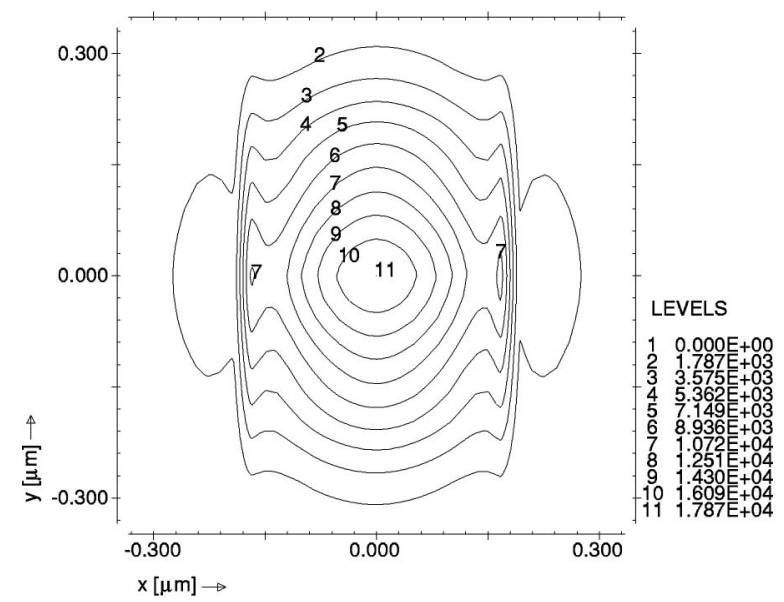

(b)

Fig. 10. Absorbed energy distributions $\left(10^{4} \mathrm{~W} / \mu \mathrm{m}^{3}\right)$ halfway in the PC layer for a TE-polarized spot that is incident on the grooved multilayer of Fig. 6. (a) Center of the spot is in the groove, (b) center of the spot is on the land.

multiplication by the factor $\exp \left(i k_{y}^{j} \nu \Delta y\right)$. This procedure is carried out for all $k_{y}^{j}$, and, by coherent summation of all these extended fields, the total EM field in all cells of interest in a neighborhood of the spot is obtained.

We assume that, in those layers of the recording stack that are absorbing, the absorbed EM energy in $\Lambda_{\text {opt }}$ is transformed entirely into heat. Hence the rate $Q\left(\mathrm{~J} \mathrm{~m}^{-3}\right)$ at which heat is generated per unit volume, owing to the absorption of light, is given by

$$
Q(\mathbf{r})=\frac{1}{2} \omega \epsilon_{0} \operatorname{Im}[\epsilon(\mathbf{r})]\left|\mathbf{E}^{\operatorname{tot}}(\mathbf{r})\right|^{2},
$$

where $\operatorname{Im}[\epsilon(\mathbf{r})]$ denotes the imaginary part of the relative electric permittivity at position $\mathbf{r}$.

\section{THERMAL MODEL}

The use of a $2 \frac{1}{2} \mathrm{D}$ optical model has the limitation that changes in the optical properties of the medium and thus in the absorbed energy distribution, owing to the heating of the laser spot, cannot be taken into account in the 3D thermal model because these changes are 3D. The effect 
of these changes on the light distribution in the stack and, in particular, on the absorbed energy is, in general, small.

The laser spot is contiguously turned on between $t$ $=0$ and $t=t_{e}$. The disk rotates at the speed $v$ (meters per second). In the thermal model a 3D computational box $\Lambda_{\text {temp }}$ with dimensions $\left(L_{x}, L_{y}, L_{z}\right)$ is used. The chosen coordinate axes are parallel to the axes of the coordinate system used in the optical model but, in contrast to the latter, are attached to the disk. Hence the coordinate system used in the heat problem moves with speed $v$ along the grooves in the positive $y$ direction with respect to the coordinate system of the optical model. In the $x$ direction the computational box $\Lambda_{\text {temp }}$ consists of a grooved structure of equal width as the extended region $\Lambda_{\text {opt }}$ of the optical model. This structure is further extended ungrooved to the exterior of $\Lambda_{\text {temp }}$.

The temperature rise above ambient in the disk, $T(\mathbf{r}, t)$ (degrees Celsius), satisfies the heat diffusion equation:

$$
\rho C \frac{\partial T}{\partial t}-\nabla \cdot[\kappa \nabla T]=Q(x, y+v t, z),
$$

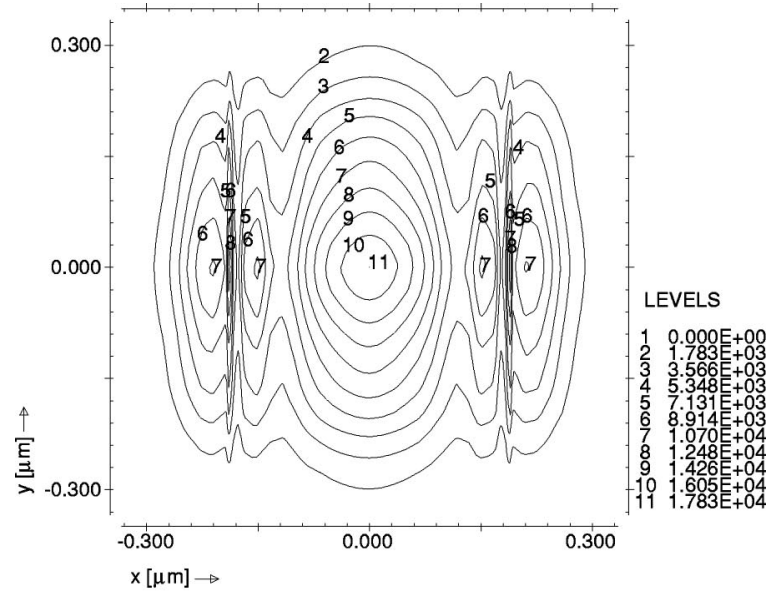

(a)

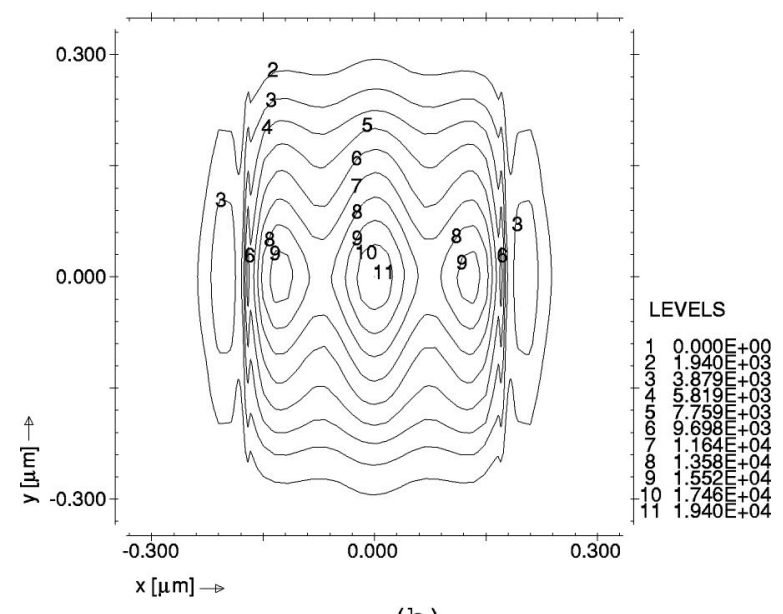

(b)

Fig. 11. Absorbed energy distributions $\left(10^{4} \mathrm{~W} / \mu \mathrm{m}^{3}\right)$ halfway in the PC layer for a TM-polarized spot that is incident on the grooved multilayer of Fig. 6. (a) Center of the spot is in the groove, (b) center of the spot is on the land.
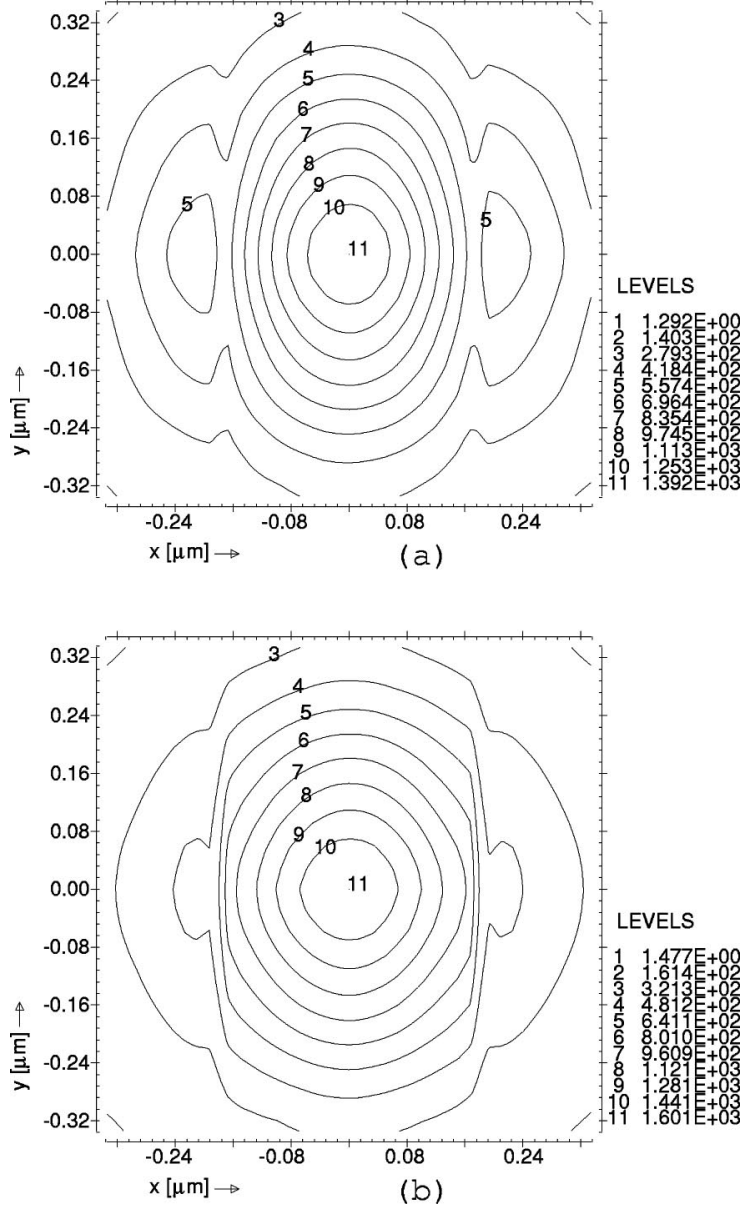

Fig. 12. Temperature distributions $\left({ }^{\circ} \mathrm{C}\right)$ halfway in the PC layer for a TE-polarized spot that is incident on the grooved multilayer of Fig. 6. (a) Center of the spot is in the groove, (b) center of the spot is on the land. (Temperatures should be corrected for latent heat.)

where $\kappa\left(\mathrm{W} / \mathrm{m}^{\circ} \mathrm{C}\right)$ is the heat conductivity, $\rho\left(\mathrm{kg} / \mathrm{m}^{3}\right)$ is the density, and $C\left(\mathrm{~J} / \mathrm{kg}^{\circ} \mathrm{C}\right)$ is the specific heat. These quantities are in general different in different layers of the optical disk but are taken constant in each layer. Hence they are assumed to be independent of temperature in the temperature range of interest. Note that by choosing the coordinate system to move with the disk, no additional convection term is introduced, but the source term becomes time dependent. The heat problem is taken to be adiabatic, and therefore we take as boundary condition

$$
\partial T / \partial n=0
$$

where $n$ denotes the outward-pointing unit normal at any of the outer surfaces of $\Lambda_{\text {temp. }}$. The dimensions of $\Lambda_{\text {temp }}$, in particular the lengths of the ungrooved continuations in the $x$ direction, are chosen large enough to ensure that the choice of boundary conditions does not influence the solution.

In Eq. (24) it is assumed that the temperature rises because of the total generated heat. In fact, a considerable fraction of $Q$ is latent heat, which does not contribute to the rise in temperature but instead is used for the solidto-liquid transition during melting of the PC material. 
The diffusion problem [Eqs. (24) and (25)] is 3D and time dependent. It could, in principle, be reduced to a set of uncoupled time-dependent problems on the unit cell $\Omega$ by Fourier expanding $Q$ and $T$ in terms of functions that are periodic in $x$ apart from a phase shift, as was done for the optical problem. But since the diffusion problem is a BVP for a single real-valued function, the time-dependent 3D problem can just as well be efficiently solved directly on the $3 \mathrm{D}$ region.

On the region $\Lambda_{\text {temp }}$ a mesh is generated, consisting of tetrahedral elements whose size increases toward the edges; see Fig. 4. The coarseness is taken such that, in the subregion $\Lambda_{\text {opt }}$ of $\Lambda_{\text {temp }}$, the fineness of the tetrahedral mesh is approximately of the same order in size as that of the triangular mesh used in the EM field calculation. In points of $\Lambda_{\mathrm{opt}}$ the absorbed energy $Q$ is mapped onto the mesh for $\Lambda_{\text {temp }}$ by means of a linear interpolation; see Fig. 5. Each nodal point of the tetrahedral grid is first interpolated within the corresponding triangles of two of the expanded cells in planes differing by the distance $\Delta y$ of the optical mesh, by use of linear basis functions. The obtained interpolated values are then once more linearly interpolated in the $y$ direction to find the
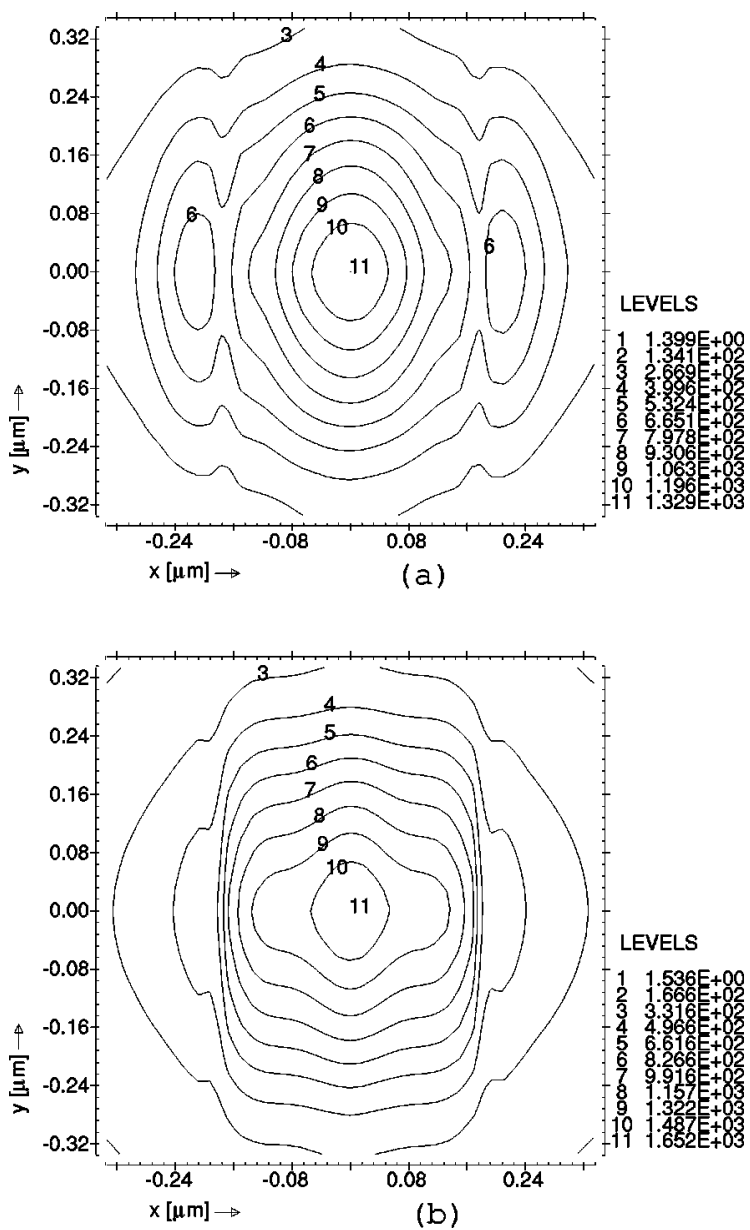

Fig. 13. Temperature distributions $\left({ }^{\circ} \mathrm{C}\right)$ halfway in the $\mathrm{PC}$ layer for a TM-polarized spot that is incident on the grooved multilayer of Fig. 6. (a) Center of the spot is in the groove, (b) center of the spot is on the land. (Temperatures should be corrected for latent heat.)
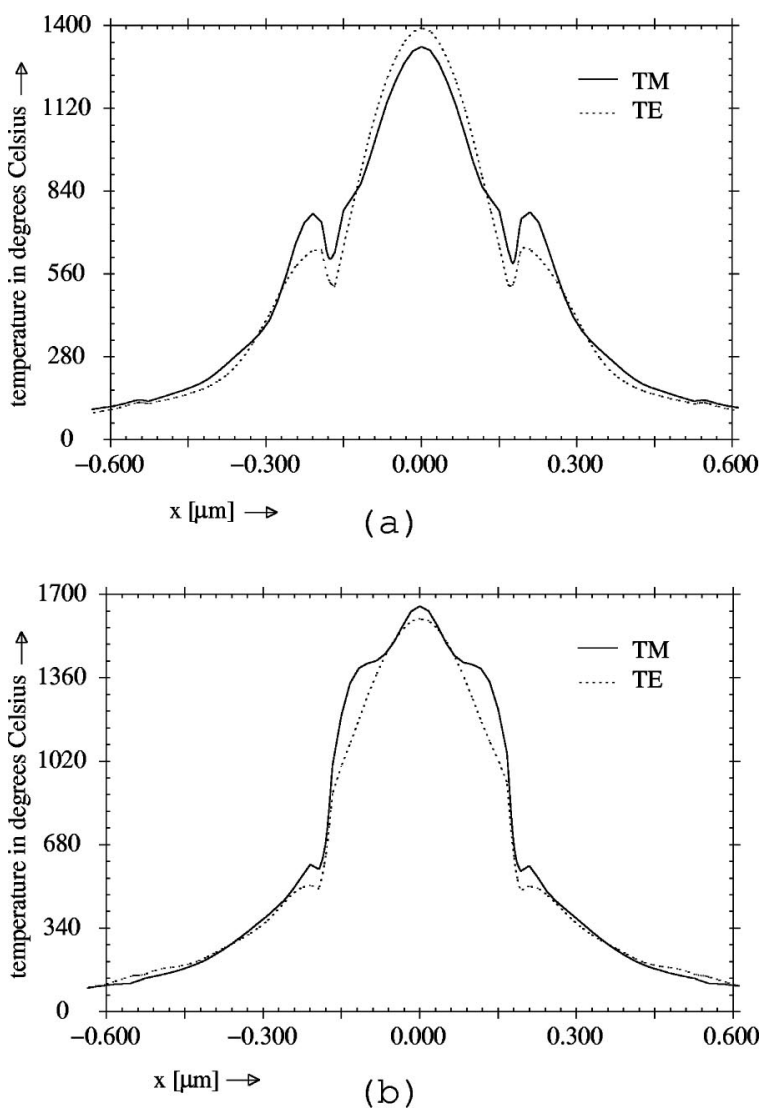

Fig. 14. Cross sections of the temperature distributions $\left({ }^{\circ} \mathrm{C}\right)$ halfway in the PC layer as shown in Figs. 12 and 13. (a) Center of the spot is (a) in the groove, (b) on the land. (Temperatures should be corrected for latent heat.)

absorbed energy in the point of interest. In points of $\Lambda_{\text {temp }}$ outside of $\Lambda_{\text {opt }}$, the absorbed energy is taken equal to zero.

The temperature problem [Eqs. (24) and (25)] is solved by use of the FEM with piecewise linear basis functions. The finite linear system of equations is, for every time step, iteratively solved with the conjugate gradient method. For the time integration the Euler implicit method is used.

\section{NUMERICAL RESULTS}

The model described in the preceding sections has been applied to two land-groove configurations. The first configuration is chosen equal to the multilayered stack called "case C1" by Peng and Mansuripur ${ }^{5}$ and is shown in Fig. 6.

The optical axis coincides with the $z$ axis and is in the middle of Fig. 6. Hence the center of the spot is at the center of the groove in Fig. 6, and therefore this case is called groove recording. When the stack is translated over half a period in the $x$ direction, the center of the spot is at the center of the land, and therefore this situation is called land recording. In all simulations the spot is focused in air in the middle of the PC layer, and the disk is at rest.

The track pitch is $720 \mathrm{~nm}$. The groove depth of $50 \mathrm{~nm}$ has been exaggerated in order to study to what extent the 
polarization of the incident spot influences the absorbed energy and the temperature distribution. The material properties of the stack are listed in Table 1 . The beam that is incident on the aperture of the lens is Gaussian, having its $1 / e$ amplitude at the rim of the aperture of the lens. The output power of the laser is $4 \mathrm{~mW}$, of which $86 \%$ is captured by the objective lens. The lens has a $\mathrm{NA}=0.65$ and wavelength of the incident light $\lambda$ $=410 \mathrm{~nm}$.

Before studying a grooved stack, we consider the interaction of a focused spot with an ungrooved version of the multilayer stack. The results of the simulations for this configuration are shown in Figs. 7 and 8. In Fig. 7 the amplitude of the dominant magnetic field component and the absorbed energy distributions are shown for a TMpolarized spot, i.e., a spot for which the electric field is predominantly parallel to the $x$ direction. The layers shown in the figures are (from bottom to top) the reflective layer, the first dielectric layer, the PC layer, and, finally, the second dielectric layer. In Fig. 7(a) standing waves are clearly visible. It follows from Fig. 7(b) that almost all the light is absorbed by the PC layer.
In Fig. 8(a) the absorbed energy distribution is shown halfway in the PC layer $(z=144 \mathrm{~nm})$ after the extension of the 2D distribution shown in Fig. 7(b) and a mapping to the $3 \mathrm{D}$ thermal mesh. Figure 8 (b) shows the corresponding temperature distributions halfway in the PC layer for TM polarization after $t=100 \mathrm{~ns}$.

Because the multilayer has flat interfaces, the absorbed energy density and temperature distribution in the planes $z=$ constant corresponding to TE polarization are, of course, obtained from those of TM polarization by a counterclockwise rotation over $90 \mathrm{deg}$ around the $z$ axis.

In Fig. 9(a) the temperature distribution is shown in the $y=0$ plane when a TM-polarized spot is incident on the flat multilayer (the $y=0$ plane contains the center of the spot). Figure $9(\mathrm{~b})$ shows the temperature along the line $x=y=0$ as a function of the depth coordinate $z$. It can be seen that there are steep gradients near and within the PC layer. This implies that slightly changing the position of the intersection plane can lead to relatively large differences in the temperature profile. This is important when one compares the results with those found

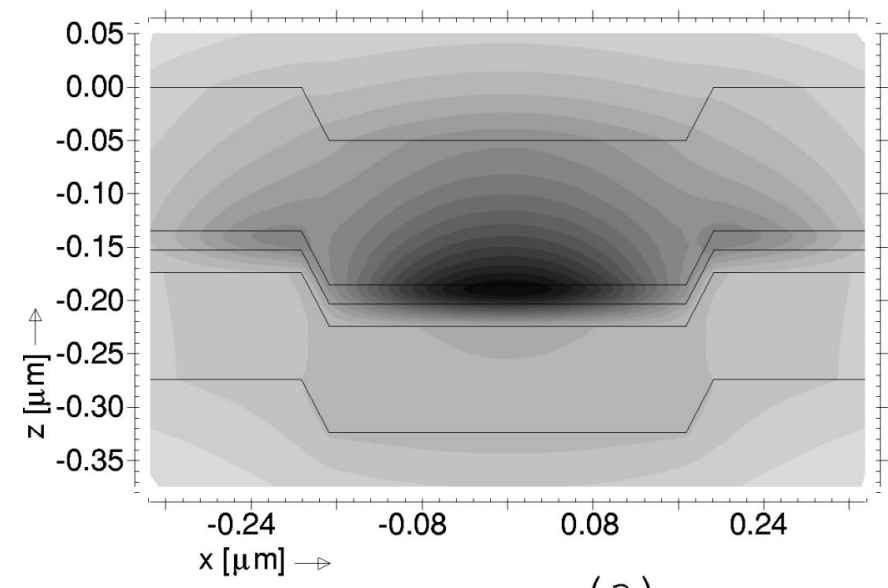

(a)

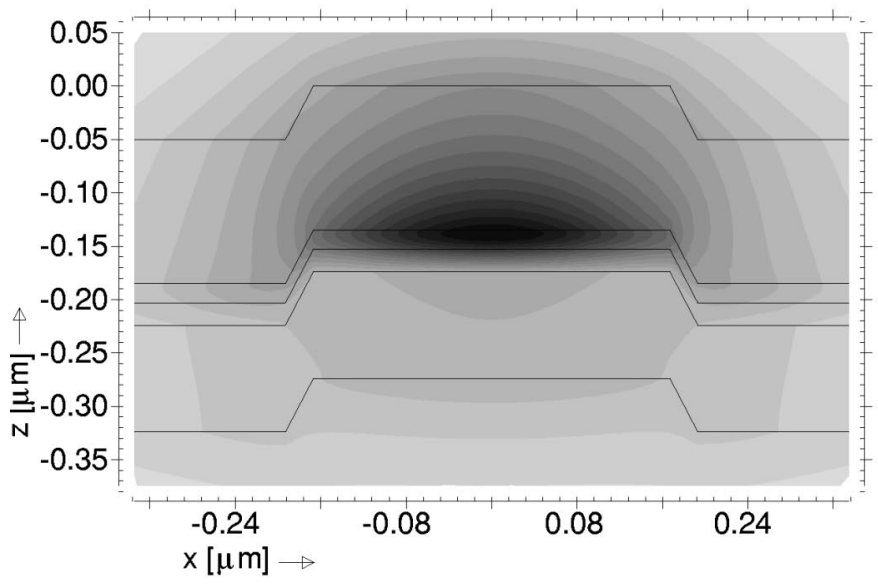

(b)

\section{LEVELS}

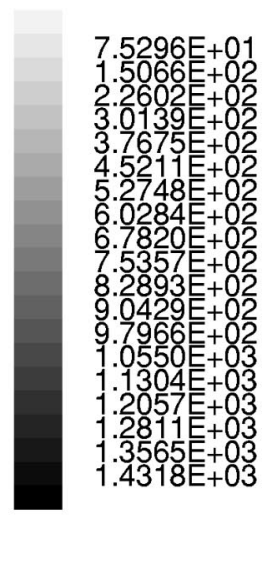

LEVELS

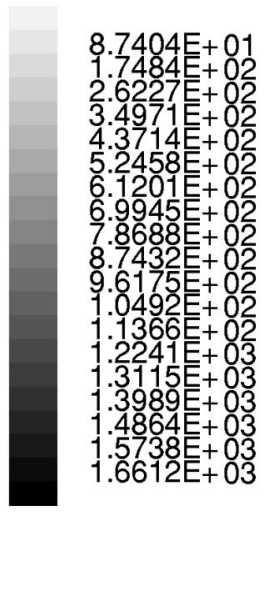

Fig. 15. Temperature distribution $\left({ }^{\circ} \mathrm{C}\right)$ for a TE-polarized spot in a cross section perpendicular to the groove direction for the stack of Fig. 6. (a) For groove recording, (b) for land recording. (The temperature values are much too large because the effect of latent heat is neglected). 


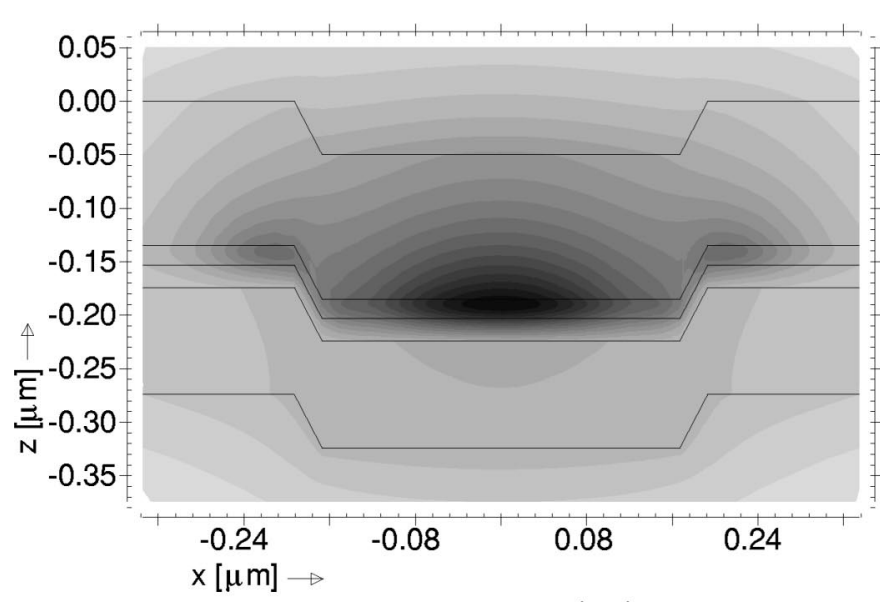

(a)

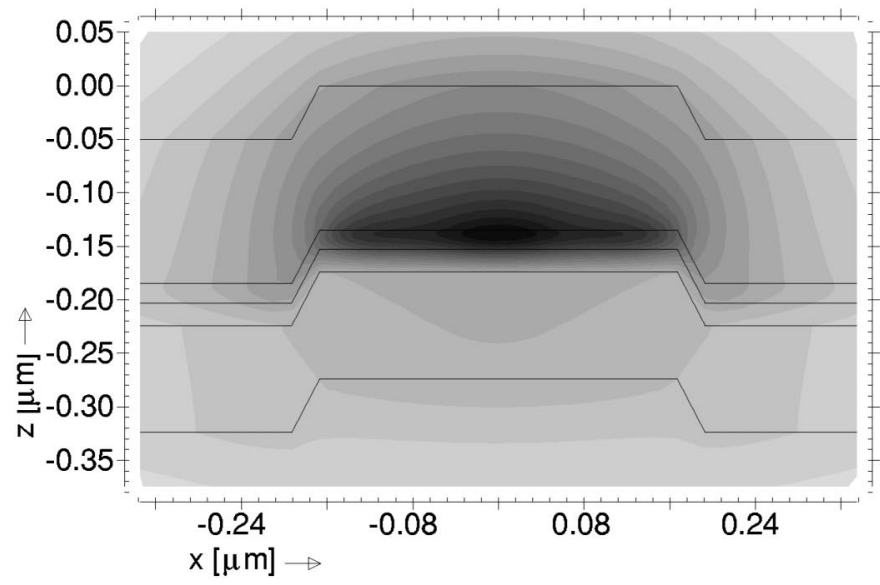

(b)
LEVELS

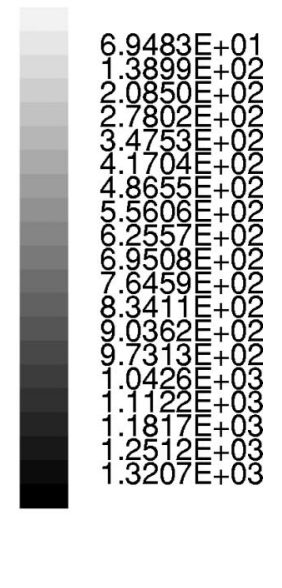

LEVELS

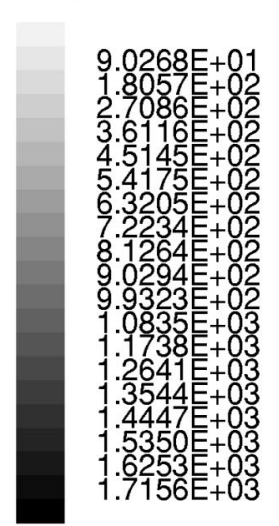

Fig. 16. Temperature distribution $\left({ }^{\circ} \mathrm{C}\right)$ for a TM-polarized spot in a cross section perpendicular to the groove direction for the stack of Fig. 6. (a) For groove recording, (b) for land recording. (The temperature values are much too large because the effect of latent heat is neglected).

elsewhere in the literature. It should be remarked that in this and in the other figures, the temperatures shown are unrealistically large owing to neglect of the effect of latent heat.

In Fig. 10, cross sections through the middle of the PC layer are shown for the absorbed energy distributions for a TE-polarized spot that is focused on a groove [Fig. 10(a)] and a land [Fig. 10(b)] of the configuration shown in Fig. 6. The cross-sectional planes follow the topography of the grooves. In Fig. 11 the analogous results are shown for a TM-polarized spot. The corresponding temperature distributions after the spot has been turned on for $100 \mathrm{~ns}$ are shown in Figs. 12 and 13 (these are also cross sections through the middle plane of the PC layer).

Noticeable differences can be observed according to whether the spot is focused on a land or a groove and also between different polarizations. For the absorbed energy of the TE-polarized spot, a much smoother profile is found along the edges of the groove than for a TM-polarized spot. This holds for both the land- and the groovecentered spots. Also, the TM case shows considerably
Table 2. Numerical Values for the Complex Refractive Index $n$, Heat Capacity $\rho C$, and Thermal Conductivity $\kappa$ as Used in the Simulations of the Blu-Ray Disc

\begin{tabular}{lccl}
\hline Material & $n$ & $\begin{array}{c}\rho C \\
\left(\mathrm{~J} / \mathrm{cm}^{3}{ }^{\circ} \mathrm{C}\right)\end{array}$ & $\begin{array}{c}\kappa \\
\left(\mathrm{W} / \mathrm{cm}{ }^{\circ} \mathrm{C}\right)\end{array}$ \\
\hline Cover & 1.55 & 1.75 & 0.0025 \\
PC layer $\left(\mathrm{Ge}_{6} \mathrm{In}_{2} \mathrm{Sb}_{72} \mathrm{Te}_{20}\right)$ & $1.5+3.45 i$ & 1.5 & 0.05 \\
Dielectric $\left(\mathrm{ZnS}-\mathrm{SiO}_{2}\right)$ & 2.3 & 2.005 & 0.0058 \\
$\mathrm{Si}_{3} \mathrm{~N}_{4}$ & 2.07 & 1.7 & 0.08 \\
Reflector (Ag) & $0.173+1.999 i$ & 1.9 & 0.7 \\
Substrate (polycarbonate) & 1.62 & 1.7 & 0.0023 \\
\hline
\end{tabular}

steeper gradients on and near the edges. Besides, a larger number of local maxima are found for the TMpolarized spot.

In Fig. 14 the cross-track temperature distributions in the middle of the PC layer and parallel to the $x$ axis are shown for both polarizations when the focused spot is po- 


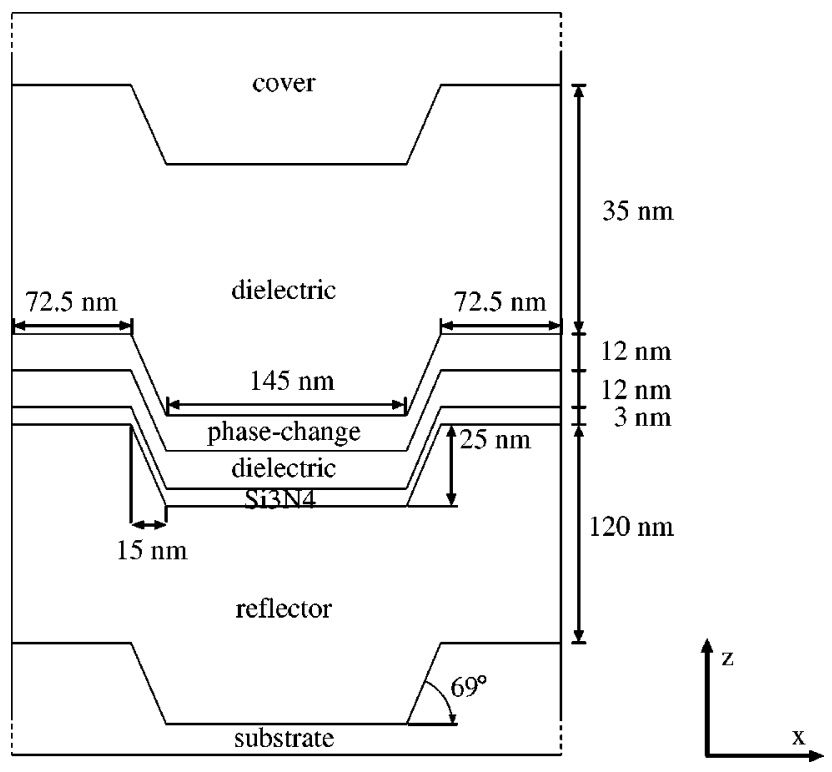

Fig. 17. Cross section of the stack as used for the Blu-ray Disc simulation.

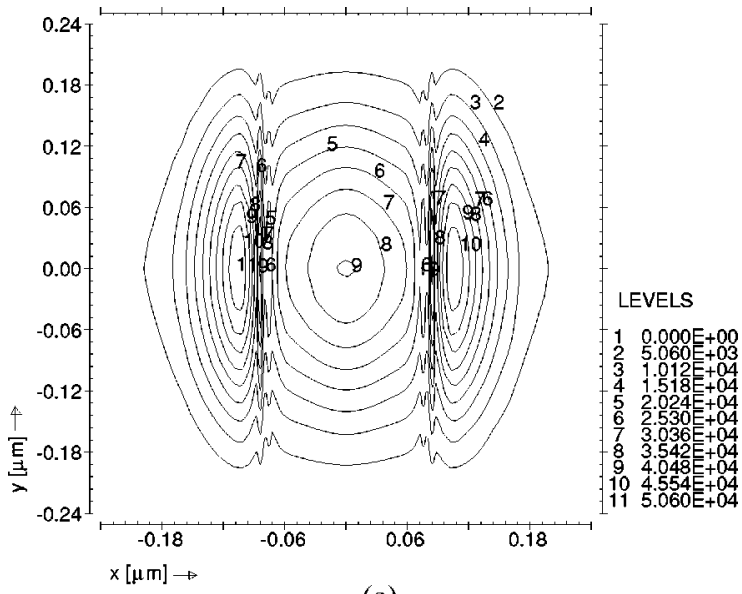

(a)

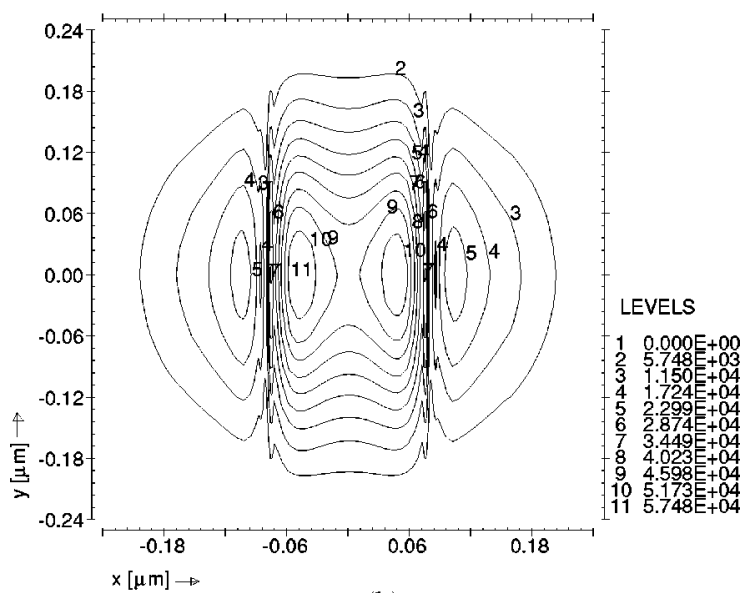

(b)

Fig. 18. Absorbed energy distributions $\left(10^{4} \mathrm{~W} / \mu \mathrm{m}^{3}\right)$ halfway in the PC layer for a TM-polarized spot that is incident on the grooved multilayer for the Blu-ray Disc (stack is shown in Fig. 15). The NA of the lens is 0.85 , the wavelength of the light is $405 \mathrm{~nm}$. (a) Center of the spot is in the groove, (b) center of the spot is on the land.

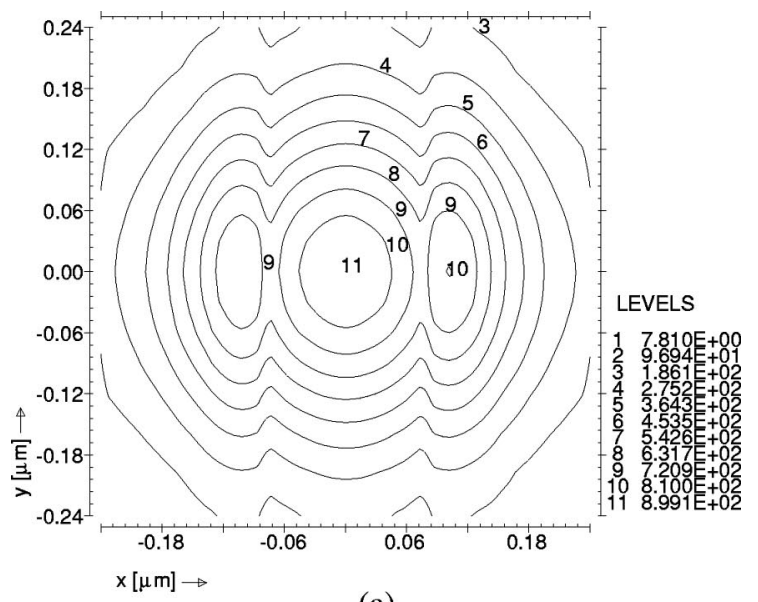

(a)

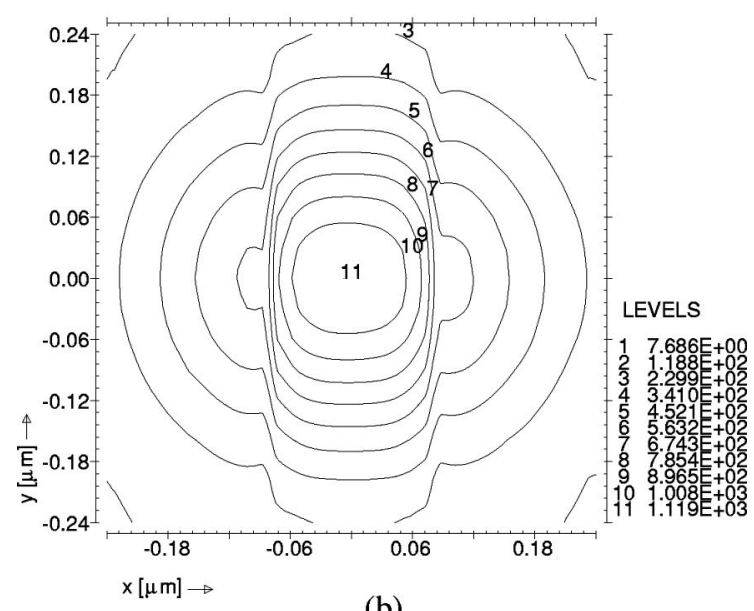

(b)

Fig. 19. Temperature distributions $\left({ }^{\circ} \mathrm{C}\right)$ halfway in the PC layer for a TM-polarized spot that is incident on the grooved multilayer for the Blu-ray Disc of Fig. 15. (a) Center of the spot is in the groove, (b) center of the spot is on the land. (Temperatures should be corrected for latent heat.)

sitioned over either a land or a groove. The sidelobes in the temperature distributions seem to be typical for TMpolarized spots. When positioned over a land, both polarizations show steeper gradients near the edges of the groove. When the spot is positioned over a groove, temperatures found on the neighboring lands are higher than temperatures found on the neighboring grooves when the spot is positioned over a land. These higher temperatures of $\approx 650^{\circ} \mathrm{C}$ imply a possible occurrence of undesirable cross-track cross-talk effects.

The shape of the isotherms as shown in Figs. 12 and 13 and the cross-track temperature distributions of Fig. 14 are very similar to the corresponding results published by Peng and Mansuripur. ${ }^{5}$ Absolute temperatures differ, however. The reason is that in the current work the latent heat, which is used for the solid-to-liquid transition during melting, is not taken into account. The temperatures as shown in the figures should therefore be corrected for this latent heat.

In Figs. 15 and 16, temperature distributions are shown in the plane $y=0$ that is perpendicular to the grooves and contains the center of the spot. In Fig. 15 the spot is TE polarized, and in Fig. 16 it is TM polarized. 
It can be seen that the temperature in the dielectric above the PC layer is quite high.

Finally, we consider the Blu-ray Disc. The numerical values of the physical parameters of the various materials are listed in Table 2. In Fig. 17 a cross section of one period of the multilayer stack of the Blu-ray Disc is shown. The track pitch is only $320 \mathrm{~nm}$. It can be seen that, compared with the stack in Fig. 6, there is an additional thin layer above the reflector. Furthermore, the PC material and the reflector consist of materials other than in the previous example. The wavelength is $\lambda=405 \mathrm{~nm}$. The output power of the laser is $4 \mathrm{~mW}$, as before. The focusing lens now has NA $=0.85$. The spot is again focused in air in the middle of the PC layer. The plane wave front that is focused by the lens is linearly polarized with the electric field vector parallel to the $x$ direction. Therefore the field of the spot is predominantly TM polarized. Because of the high NA, it is now essential to take the rotation of polarization into account when the light is focused by the lens. As has been remarked before, the spots that we use as an incident field have all been calculated by applying the theory of Ignatowsky ${ }^{11}$ and Richards and Wolf. $^{12}$

In Figs. 18 and 19 the absorbed energy and temperature distributions after $100 \mathrm{~ns}$ are shown in the plane in the middle of the PC layer of the Blu-ray Disc (the crosssectional plane follows the topography of the grooves). When the results for groove and land recording in Figs. 18(a) and 18(b) are compared with the absorbed energy distributions in Fig. 11 (for which the incident spot is also predominantly TM polarized), quite large differences can be seen. First of all, owing to the higher NA of the lens, the spots are narrower in the Blu-ray case. Furthermore, the distributions for the Blu-ray stack have fewer local extrema. Besides, for the Blu-ray case the highest absorption values are observed near, or even on, the sloped edges, instead of in the center of the groove or land.

Although the absorption values for the Blu-ray stack are more than twice as large as those found for the stack of Fig. 6, the corresponding temperature profiles shown in Fig. 19 show considerably lower values. The shape of the

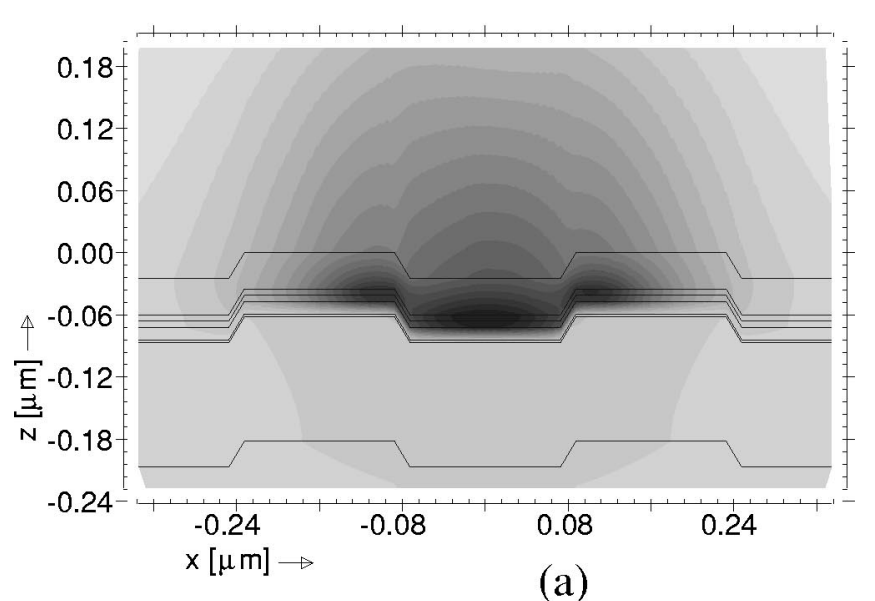

(a)

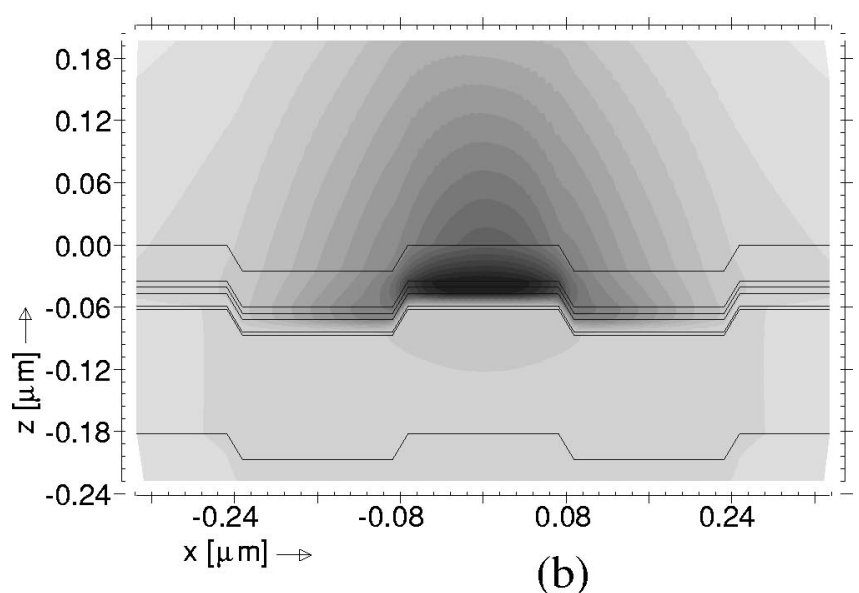

\section{LEVELS}

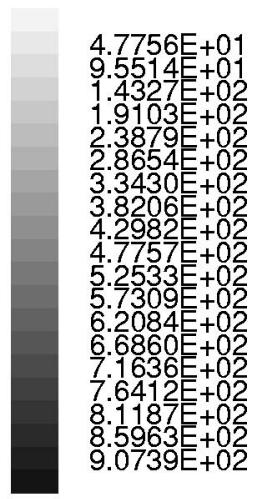

\section{LEVELS}

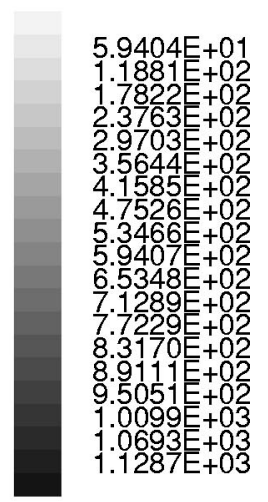

Fig. 20. Temperature distribution $\left({ }^{\circ} \mathrm{C}\right)$ for a TM-polarized spot in a cross section perpendicular to the groove direction for the Blu-ray stack of Fig. 15. (a) Center of the spot is in the groove, (b) center of the spot is on the land. (Temperatures should be corrected for latent heat. 
isotherms, however, is similar to that observed in Fig. 13. The lower temperatures in the case of the Blu-ray stack are caused by the fact that the illuminated region of the $\mathrm{PC}$ layer is smaller because the spot is narrower and the $\mathrm{PC}$ layer is thinner.

In Fig. 20, cross sections are shown of the temperature in the plane $y=0$ (this plane contains the center of the spot). The highest temperatures occur for land recording.

\section{CONCLUSIONS}

The $2 \mathrm{D}$ rigorous diffraction model for the scattering of a $3 \mathrm{D}$ incident spot on a $2 \mathrm{D}$ periodically grooved geometry by Brok and Urbach ${ }^{7}$ has been successfully modified to obtain the total EM field in a 3D region of interest for optical recording simulations. The optical model has been combined with a 3D thermal model in order to compute the temperature distribution in a grooved multilayer recording stack. The results are found to be in good accordance with results published by Peng and Mansuripur. ${ }^{5}$ Furthermore, results were presented for groove and land recording on a Blu-ray Disc.

\section{ACKNOWLEDGMENT}

This research is supported by the Technology Foundation STW, applied science division of the Nederlandse Organisatie voor Wetenschappelijk Onderzoek (NWO) (Dutch Organization for Scientific Research) and the technology program of the Ministry of Economic Affairs.

The authors can be reached by e-mail at j.h.brusche@ewi.tudelft.nl, a.segal@ewi.tudelft.nl, and h.p.urbach@philips.com.

\section{REFERENCES AND NOTES}

1. J. B. Judkins and R. W. Ziolkowski, "Finite-difference timedomain modeling of nonperfectly conducting metallic thinfilm gratings," J. Opt. Soc. Am. A 12, 1974-1983 (1995).

2. W.-C. Liu and M. W. Kowarz, "Vector diffraction from subwavelength optical disk structures: two-dimensional modeling of near-field profiles, far-field intensities, and detector signals from a DVD," Appl. Opt. 38, 3787-3797 (1999).

3. D. S. Marx and D. Psaltis, "Optical diffraction of focused spots and subwavelength structures," J. Opt. Soc. Am. A 14, 1268-1278 (1997).

4. W.-H. Yeh, L. Li, and M. Mansuripur, "Vector diffraction and polarization effects in optical disk systems," Appl. Opt. 37, 6983-6988 (1998).

5. C. Peng and M. Mansuripur, "Thermal cross-track cross talk in phase-change optical disk data storage," J. Appl. Phys. 88, 1214-1220 (2000).

6. Y. Nishi, T. Shimano, H. Kando, M. Terao, and T. Maeda, "Simulations of marks formed on phase-change, land/ groove disks," Jpn. J. Appl. Phys. 41, 2931-2938 (2002).

7. J. M. Brok and H. P. Urbach, "Rigorous model of the scattering of a focused spot by a grating and its application in optical recording," J. Opt. Soc. Am. A 20, 256-272 (2003).

8. SEPRAN is a finite-element package of SEPRA (Ingenieursbureau), Den Haag, The Netherlands (www.sepra.nl).

9. H. P. Urbach and R. T. M. Merkx, "Finite element simulation of electromagnetic plane wave diffraction at gratings for arbitrary angles of incidence," G. Cohen, L. Halpern, and P. Joly, eds., in Mathematical and Numerical Aspects of Wave Propagation Phenomena, Proceedings of SIAM First Conference on Mathematical and Numerical Aspects of Wave Propagation (Society for Industrial and Applied Mathematics, Philadelphia, Pa., 1992), pp. 89-99.

10. DIFFRACT is a product of MM Research, Inc., Tucson, Ariz. Its theoretical basis has, e.g., been described by M. Mansuripurin, "Certain computational aspects of vector diffraction problems," J. Opt. Soc. Am. A 6, 786-805 (1989).

11. V. S. Ignatowsky, "Diffraction by an objective lens with arbitrary aperture," Trans. Opt. Inst. Petrograd 1, 1-36 (1921).

12. B. Richards and E. Wolf, "Electromagnetic diffraction in optical systems: II. Structure of the image in an aplanatic system," Proc. R. Soc. London Ser. A 253, 358-379 (1959). 